\title{
A De Rham-Witt approach to crystalline rational homotopy theory
}

\author{
Minhyong Kim and Richard M. Hain
}

\begin{abstract}
We give a definition of the crystalline fundamental group of suitable log schemes in positive characteristic using the techniques of rational homotopy theory applied to the De Rham-Witt complex.
\end{abstract}

\section{Introduction}

Throughout this paper, $k$ will denote a perfect field of characteristic $p>0, W_{n}=W_{n}(k)$ the ring of Witt vectors of $k$ of length $n$, and $W=\lim W_{n}$. In addition, $K$ denotes the fraction field of $W$.

We wish to present a relatively direct approach to the unipotent crystalline fundamental group of a variety over $k$ using the De Rham-Witt (DRW) approach [Blo77, HK94, Hyo91, Hyo88, Ill79] and constructions that come from rational homotopy theory [Che77, Hai87a, Hai87b, Mor78, Nav87, Qui69, Sul77]. In the process, to a smooth connected proper fine $\log$ scheme $Y$ over $k$ of Cartier type, we will associate a canonical commutative differential graded algebra that deserves to be called the unipotent crystalline rational homotopy type. The zeroth cohomology of the bar construction on this algebra will then give us the coordinate ring of the unipotent crystalline fundamental group. The use of the DRW complex allows us to easily endow the crystalline fundamental group with natural expected structures such as Frobenius and monodromy operators and, importantly, a weight filtration.

In the influential paper [Del89], Deligne outlined a motivic theory of the fundamental group. Given a variety $V$ defined over a number field $F$, one should have a unipotent algebraic fundamental group corresponding to each cohomology theory associated to $V$, Betti, étale, De Rham, and crystalline, together with suitable comparison isomorphisms between them. Following up on this idea, Deligne gave a Tannakian definition of a crystalline fundamental group using the theory of the De Rham fundamental group for a variety with good reduction. That is, if $v$ is a prime of $F$, the De Rham fundamental group over $F_{v}$ together with a Frobenius action was taken to define the crystalline fundamental group of the special fiber. This definition was reasonable in the context of Deligne's paper since he was primarily interested in varieties over number fields and, of course, one could have started from a variety defined over $F_{v}$ in this construction. This corresponds to the viewpoint that for a variety over $F_{v}$, the crystalline cohomology is just 'extra structure' on De Rham cohomology. However, it is evident that this definition is not quite satisfactory. The most important problem is that the crystalline fundamental group is not defined intrinsically for a variety in positive characteristic. Another problem is to deal with the case of bad reduction.

In [CLS99, Shi00, Shi02], Chiarellotto and Le Stum, and independently Shiho, gave an intrinsic definition of a crystalline fundamental group $\pi_{1}^{\mathrm{cr}}$ of a proper $\log$ smooth variety over a perfect field

Received 11 February 2002, accepted in final form 6 June 2003.

2000 Mathematics Subject Classification 14G40.

Keywords: De Rham-Witt complex, bar complex, crystalline cohomology.

This journal is (C) Foundation Compositio Mathematica 2004. 


\section{KIM AND R. M. HAIN}

of positive characteristic $p$ which is equipped with a comparison isomorphism to $\pi_{1}^{\mathrm{dr}}$ in a 'lifted' setting. In Shiho, for example, the crystalline fundamental group was defined as the fundamental group of the category of unipotent isocrystals, and the comparison theorem was effected through the intermediary of a 'convergent' fundamental group, interesting in its own right.

In the present paper, we use an approach to the crystalline fundamental group suggested by the work of Wojtkowiak [Woj93] where the De Rham rational homotopy groups were constructed over an arbitrary ground field using cosimplicial schemes. Shiho's announcement [Shi98] also indicates a similar approach using a category of 'complexes' of schemes. The idea of both authors is to construct a model for path space purely in the context of algebraic geometry out of which one can extract the homotopy groups via cohomological techniques.

On the other hand, we exploit the fact that a formal algebraic model already exists for the cohomology of path space, namely, the bar construction, provided one has a commutative differential graded algebra (CDGA) which is a 'purely algebraic' analogue of the smooth differential forms. The problem here, as in rational homotopy theory, is that the usual resolutions which one uses to compute various cohomology groups of sheaves of CDGAs do not give rise to a CDGA in general, even though they are equipped with a multiplication which is homotopy commutative. However, it turns out that a CDGA model with the right properties is available from the work of Navarro-Aznar [Nav87] (already used in [Woj93]), where a derived 'Thom-Whitney' functor is constructed that associates in a canonical fashion a CDGA to a sheaf of CDGAs on a topological space. Although the setting for Navarro's work is the Hodge theory of complex varieties, it is clear that there is actually a powerful technique that applies to quite general topoi underlying his constructions. More precisely, all one needs to apply his machinery is a topos with enough points.

The CDGA we use for our definition is the algebra of De Rham-Witt (DRW) differential forms, considered as a pro-sheaf on the small étale site of a variety. This is very natural since, in many senses, the DRW differentials are the 'correct' analogue of algebraic differential forms suitable for crystalline constructions. This approach has the added advantage that the definition of $\pi_{1}^{\text {cr }}$ is quite elementary and the comparison with the De Rham fundamental group requires only existing cohomological techniques.

But perhaps the main interest in the approach we present is the natural definition of a weight filtration on the cohomology of the bar construction, and hence, on the coordinate ring and the Lie algebra of the crystalline fundamental group. For this, we use a variant of the Hyodo-Steenbrink complex introduced by Mokrane [Mok93], modeled on the complex underlying the "limit mixed Hodge structure' for homotopy groups [Hai87b].

Referring to the following sections for precise terminology, we state now the main theorems of the paper.

Let $Y$ be a connected proper smooth fine log scheme over $k$ of Cartier type, where $k$ is endowed with some fine log structure, and let $W \omega_{Y}$ be the pro-sheaf of CDGAs consisting of the De RhamWitt differential forms of Illusie-Hyodo-Kato.

The crystalline rational homotopy type $A_{Y}$ of $Y$ is defined by the following formula:

$$
A_{Y}=s_{\mathrm{TW}} \lim _{K} \Gamma\left(G\left(W \omega_{Y}\right)\right) \text {. }
$$

The notation, which will be explained below in detail, is that given a pro-sheaf $L, G(L)$ is its canonical cosimplicial Godement resolution, $\lim _{K}$ is the operation which associates to an inverse system of (cosimplicial) $W_{n}$-modules the (cosimplicial) $W$-module obtained by taking the inverse limit, and then forms a tensor of it with $K$, and finally, $s_{\mathrm{TW}}$ is Navarro-Aznar's 'simple Thom-Whitney algebra' functor. A choice of a point $y$ determines an augmentation for $A_{Y}$, and we can form the bar complex, which we denote by $B(Y, y)$. Then $C r(Y, y):=H^{0}(B(Y, y))$ has the natural structure of a 


\section{CRYSTALline RATIONAL HOMOTOPY THEORY}

commutative Hopf algebra filtered by finitely generated Hopf algebras. The crystalline fundamental group is defined by

$$
\pi_{1}^{\mathrm{cr}}(Y, y)=\operatorname{Spec} C r(Y, y) .
$$

When $k$ is equipped with the log structure of a 'punctured point' and the point gives an exact embedding of $\log$ schemes, we will see below that $\pi_{1}^{\mathrm{cr}}(Y, y)$ is naturally equipped with a semi-linear Frobenius as well as a monodromy operator, which is interpreted as a vector field on $\pi_{1}^{\mathrm{cr}}$, and that they satisfy the usual relations.

When $Y$ is semi-stable, the weight filtration on the DRW complex induces one on the bar complex and on $C r(Y, y)$. An obvious formalism of 'mixed Frobenius complexes' then yields our main result, as follows.

TheOrem 1. Assume that $k$ is finite and that $Y$ is globally the union of smooth components that meet transversally. Then the spectral sequence for the weight filtration degenerates at $E_{2}$ and gives $\mathrm{Cr}(Y, y)$ the structure of a mixed isocrystal.

This structure is compatible with the Hopf algebra structure, and hence, also induces the structure of a mixed isocrystal on $\operatorname{Lie}\left(\pi_{1}^{\mathrm{cr}}(Y, y)\right)$.

If $X$ is a $\log$ smooth variety over a field $F$ of characteristic 0 , we associate to $X$ its De Rham rational homotopy type by the formula

$$
A_{X}=s_{\mathrm{TW}} \Gamma\left(G\left(\Omega_{X / F}\right)\right) .
$$

Given an augmentation associated to a point $x$, one then forms the bar complex $B(X, x)$ and defines the De Rham algebra

$$
D R(X, x):=H^{0}(B(X, x)) .
$$

The De Rham fundamental group is given by [Woj93]

$$
\pi_{1}^{\mathrm{dr}}(X, x)=\operatorname{Spec}(D R(X, x)) .
$$

As mentioned previously, one advantage of our approach is that Berthelot-Ogus-type comparison theorems are within the scope of crystalline cohomological techniques.

Let $A$ be a complete discrete valuation ring of mixed characteristic with fraction field $F$ and perfect residue field $k$. Let $W$ be the ring of Witt vectors of $k$ with fraction field $K$.

Theorem 2. Suppose $X$ is a proper connected smooth scheme over $A$ with a relative normal crossing divisor D. Equip $X$ with the $\log$ structure associated to the divisor $D$. Denote by $X^{*}$ the generic fiber of $X$ (with the induced $\log$ structure), $x$ a point of $X^{*}-D$ with reduction $y \in Y-D$. Then

$$
A_{X^{*}} \simeq A_{Y} \bigotimes_{K} F
$$

where the isomorphism is in the homotopy category of commutative differential graded algebras over F. Furthermore,

$$
C r(Y, y) \bigotimes_{K} F \simeq D R\left(X^{*}, x\right)
$$

as commutative Hopf algebras over $F$.

Corollary 1. With the assumptions of the theorem,

$$
\pi_{1}^{\mathrm{dr}}\left(X^{*}, x\right) \simeq \pi_{1}^{\mathrm{cr}}(Y, y) \bigotimes_{K} F .
$$




\section{KIM AND R. M. HAIN}

Although the main emphasis in this paper is on fundamental groups, the crystalline rational homotopy type can be used to define higher crystalline homotopy groups in the simply connected case. In particular, we also get some interesting consequences of the Artin-Mazur type for the higher rational homotopy groups of simply connected varieties over number fields from this comparison theorem.

Corollary 2. Let $X$ and $X^{\prime}$ be smooth proper connected varieties over a number field $F$ equipped with normal crossing divisors $D$ and $D^{\prime}$ (respectively). Let $v$ be a prime of $F$ where both $(X, D)$ and $\left(X^{\prime}, D^{\prime}\right)$ have good reduction in the sense that both varieties extend to smooth proper schemes over $\mathcal{O}_{F_{v}}$ and the divisors extend to relative normal crossing divisors. Suppose the pairs are isomorphic $\bmod v$ and both $X-D$ and $X^{\prime}-D^{\prime}$ are simply connected in any embedding of $F$ into the complex numbers. Then the higher rational homotopy groups of $X-D$ and $X^{\prime}-D^{\prime}$ are isomorphic for any embedding of $F$ into the complex numbers.

Wojtkowiak [Woj93] had earlier shown that these groups are also independent of the embedding.

One issue that is completely ignored in this paper is the comparison with the Tannakian view. That is, we do not show that the crystalline $\pi_{1}$ we define classifies unipotent isocrystals. We hope to carry out this comparison in a subsequent paper. Another topic we hope to deal with is relative completions of crystalline fundamental groups with coefficients in an $F$-isocrystal, the crystalline analogue of the completion of the fundamental group of a smooth variety relative to a variation of Hodge structure, which is considered in [Hai98]. For a related but different approach, the reader is also referred to the paper of Vologodsky [Vol01].

In a forthcoming publication, we will present a generalization of the comparison isomorphism to incorporate the 'Hyodo-Kato case' of semi-stable reduction over a ramified base. This will be achieved by using an 'infinitely twisted telescope' construction and the ideas of [Ogu95]. These ideas will also be applied to a proof of a $p$-adic analogue of Oda's good reduction criterion for curves [Oda95].

\section{Review of Hodge-De Rham theory for homotopy groups}

The unipotent De Rham fundamental group $\pi_{1}^{\mathrm{dr}}(X, x)$ of a space $X$, say with coefficients in $\mathbb{C}$, can be defined as the complex pro-unipotent completion of the usual fundamental group $\pi_{1}(X, x)$. That is, $\pi_{1}^{\mathrm{dr}}(X)$ is the initial object in the category of inverse systems of pro-algebraic unipotent groups $U$ over $\mathbb{C}$ equipped with group homomorphisms $\pi_{1}(X, x) \rightarrow U$.

Assuming the space has finite-dimensional $H^{1}$, one realization is constructed by considering the group algebra $R=\mathbb{C} \pi_{1}(X, x)$ together with the augmentation ideal $J$, and then the completion $\hat{R}$ of $R$ with respect to the augmentation ideal. $R$ naturally has the structure of a Hopf algebra induced by the comultiplication $c$ defined on the image of elements of $\pi_{1}$ by $c(g)=g \otimes g$. This comultiplication extends uniquely to $\hat{R}$ and the complex points of $U$ can be realized as the group-like elements in $\hat{R}$, i.e. $u \in \hat{R}$ such that $c(u)=u \otimes u$.

More precisely, $U$ is defined by the inverse system given by group-like elements in $\hat{R} / \hat{J}^{n}$. The Lie algebra of the De Rham fundamental group can then be realized as the primitive elements inside $\hat{R}$, i.e. those elements $t$ that satisfy $c(t)=t \otimes 1+1 \otimes t$.

Another way of understanding this construction is to consider the dual ind-Hopf algebra

$$
R^{*}:=\lim _{\longrightarrow} \operatorname{Hom}_{\mathbb{C}}\left(R / J^{n}, \mathbb{C}\right) .
$$

The remarks above correspond to the fact that $R^{*}$ is the affine coordinate $\operatorname{ring}$ of $\pi_{1}^{\mathrm{dr}}(X, x)$.

If $X$ is a smooth complex variety, $\pi_{1}^{\mathrm{dr}}(X, x)$ is also the Tannaka group of the category of unipotent vector bundles with flat connection associated to the fiber functor of evaluation at $x$. 


\section{CRYstalline RATional HOMOTOPY THEORY}

The point of view of this paper comes from the construction of the complex (or real) De Rham fundamental group via iterated integrals of differential forms. That is, if $X$ is a manifold, consider the CDGA $A_{X}$ of $C^{\infty}$ differential forms on $X$ with the augmentation $a_{x}$ given by pull-back to the point $x$.

In Chen's approach [Che77], one constructs locally constant functions on the loop space at $x$ via iterated integrals, which suffices to construct the coordinate ring. This process is conveniently formalized using the bar complex $B\left(A_{X}, a_{x}\right)$ associated to the augmented algebra. The detailed definition will be reviewed below, but we note that the essential part of the zeroth-degree term is given by

$$
\bigoplus_{s=0}^{\infty}\left(\bigotimes^{s} A_{X}^{1}\right)
$$

Given a tensor product $a_{1} \otimes \cdots \otimes a_{s}$ from this vector space, we get a function on loop space according to the rule

$$
\gamma \mapsto \int_{\gamma} a_{1} a_{2} \cdots a_{s}
$$

where the last quantity is the iterated integral defined according to the following prescription: Write $\gamma^{*}\left(a_{i}\right)=f_{i}(t) d t$. Then

$$
\int_{\gamma} a_{1} a_{2} \cdots a_{s}:=\int_{0 \leqslant t_{1} \leqslant t_{2} \leqslant \cdots \leqslant t_{s} \leqslant 1} f_{1}\left(t_{1}\right) f_{2}\left(t_{2}\right) \cdots f_{s}\left(t_{s}\right) d t_{1} d t_{2} \cdots d t_{s} .
$$

The elements of $H^{0}(B)$ then correspond to locally constant functions on the loop space.

We will see below that $B\left(A_{X}, a_{x}\right)$ also has the structure of a Hopf algebra which induces a commutative Hopf algebra structure on $H^{0}(B)$.

Chen's theorem [Che77] says that

$$
\pi_{1}^{\mathrm{dr}}(X, x)_{\mathbb{C}} \simeq \operatorname{Spec}\left(H^{0}(B)\right),
$$

provided we interpret the right-hand side suitably as a pro-algebraic group. (This is done using the bar filtration, also reviewed below.) In the simply connected case, we can also recover the higher complex homotopy groups from the higher cohomology of the bar complex.

One advantage of this approach as opposed to Sullivan's theory of minimal models is that the transparent relation between $A_{X}$ and the bar complex enables one to carry over extra structure from the differential forms naturally to the bar complex. In precise terms, the bar complex is functorial in $A_{X}$, while the minimal model is functorial only in an appropriate homotopy category. This allowed, for example, the construction of natural mixed Hodge structures on the coordinate ring of $\pi_{1}^{\mathrm{dr}}(X, x)$ [Hai87a] as well as on $\operatorname{Lie}\left(\pi_{1}^{\mathrm{dr}}(X, x)\right)$ for general varieties over $\mathbb{C}$. Equally important is the fact that the bar construction is completely algebraic, once one is given $A_{X}$. Thus, it can be built on an arbitrary CDGA over any field of characteristic zero.

\section{Algebraic prerequisites: the Thom-Whitney functor and the bar complex}

We will quickly review the definitions of [Nav87] and [Hai87a].

Given a category $T$, we denote by $\Delta T$ the category of cosimplicial objects in $T$ and by $\Delta^{+} T$ the category of augmented cosimplicial objects in $T$ [Del74].

Let $Y$ be a scheme over $k$.

Denote by $\mathcal{A}_{\mathbf{Q}}(Y)$ the category of pro-objects in the category of sheaves of (graded-)commutative differential-graded $W$-algebras (CDGAs) 'up to isogeny' on the small étale site of $Y$. So an object of $\mathcal{A}_{\mathbf{Q}}(Y)$ consists of a sequence $A=\left(A_{n}\right)_{n \geqslant 1}$, where each $A_{n}$ is a $W_{n}$-algebra and we are given 


\section{KIM AND R. M. HAIN}

transition maps $A_{n+1} \rightarrow A_{n}$ which we assume to be a surjection. Morphisms from $A=\left(A_{n}\right)$ to $B=\left(B_{n}\right)$ are given by

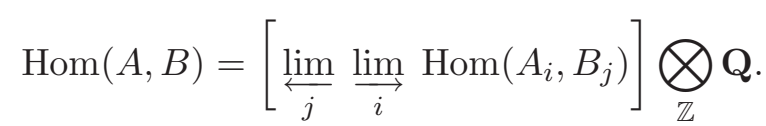

We do not distinguish notationally between the ring $W$ and the pro-sheaf $W$ regarded as an object of $\mathcal{A}_{\mathbf{Q}}(Y)$ for various $Y$. Also, the scheme $Y$ will often be suppressed from the notation if the context makes it unnecessary.

Note that an object $A$ of $\mathcal{A}_{\mathrm{Q}}$ also carries a superscript corresponding to the complex degree and an object $G$ of $\Delta \mathcal{A}_{\mathrm{Q}}$ carries two superscripts $G^{\bullet, *}$, the first denoting cosimplicial degree and the second complex degree. These superscripts will usually be suppressed from our notation. In other contexts as well, we will avoid making scripts explicit unless necessary for clarity. (In fact, it is our experience that it enhances clarity most of the time to avoid thinking about scripts.)

A word about our convention: When referring to various (pro-)sheaves with extra structure, we will often suppress the 'sheaf' in their designations unless forced upon us by considerations of clarity, and similarly we will be somewhat careless with localization up to isogeny. So an object of $\mathcal{A}_{\mathbf{Q}}(Y)$ will usually just be called a CDGA on $Y$. Also, unless explicitly stated otherwise, an algebra will refer to a $W$-algebra. On the other hand, when we put $k$ into the argument of one of our categories, such as $\mathcal{A}_{\mathbf{Q}}(k)$, we will be referring to actual objects and not sheaves. Thus, in the previous sentence, we mean the category of inverse systems of CDGAs over $W$, and not the category of sheaves on the étale site of $\operatorname{Spec}(k)$. Various constructions will be described in the sheaf case and can be modified in an obvious way for objects over $k$.

In a manner analogous to $\mathcal{A}_{\mathbf{Q}}(Y)$, we define $S h_{W, \mathbf{Q}}(Y)$, the category of pro-sheaves of $W$-modules up to isogeny, and $\mathcal{C}_{W, \mathbf{Q}}(Y)$, the category of pro-complexes of sheaves of $W$-modules up to isogeny, with similar conventions of reference as explained in the previous paragraph. Finally, $D G A_{\mathbf{Q}}(Y)$ will be the category of pro-differential graded $W$-algebras up to isogeny. Thus, $\mathcal{A}_{\mathbf{Q}}(Y)$ is a full subcategory of $D G A_{\mathbf{Q}}(Y)$ which, in turn, is equipped with a natural forgetful functor to $\mathcal{C}_{W, \mathbf{Q}}(Y)$.

Given an object $C$ of $\mathcal{C}_{W, \mathbf{Q}}$, its cohomology sheaves are by definition $\underline{H}^{i}(C):=\left(\underline{H}^{i}\left(C_{n}\right)\right)$. In what follows, of particular importance will be the situation where the $\underline{H}^{i}(C)$ are objects of $S h_{W, \mathbf{Q}}$, that is, where the transition maps are surjective. Sometimes, we will denote by $\underline{H}(C)$ the direct sum of the $\underline{H}^{i}(C)$ considered as a complex with zero differential. Thus, if $A \in \mathcal{A}_{\mathrm{Q}}$ and the transition maps for cohomology are surjective, then $\underline{H}(A)$ is naturally an object of $\mathcal{A}_{\mathbf{Q}}$ with zero differential. We will circumvent some foundational annoyances by passing to cohomology sheaves only in situations where they belong to $S h_{W, \mathbf{Q}}$. In that case, given two objects $C$ and $C^{\prime}$ of $\mathcal{C}_{W, \mathbf{Q}}$, we say they are quasiisomorphic (QI) if there is a map $f: C \rightarrow C^{\prime}$ in $\mathcal{C}_{W, \mathbf{Q}}$ which induces an isomorphism (in $S h_{W, \mathbf{Q}}$ ) of cohomology pro-sheaves. We say $A$ and $A^{\prime}$ of $\mathcal{A}_{\mathbf{Q}}$ are multiplicatively QI if they are QI as objects of $\mathcal{C}_{W, \mathbf{Q}}$ but via a map in the category $\mathcal{A}_{\mathbf{Q}}$.

The homotopy category of $\mathcal{A}_{\mathrm{Q}}$ is obtained by keeping the same objects and inverting all the multiplicative quasi-isomorphisms. We will say two objects in $\mathcal{A}_{\mathbf{Q}}$ are quasi-equivalent (QE) if they are isomorphic in the homotopy category.

Denote by $\mathbf{C}(K)$ the category of complexes of $K$ vector spaces and $\mathbf{A}(K)$ the category of CDGAs over $K$, equipped with the natural forgetful functor to $\mathbf{C}(K)$.

The functor $\lim _{K}$ from $\mathcal{C}_{W, \mathbf{Q}}(k)$ to $\mathbf{C}(K)$ takes a pro-complex of $W_{n}$-modules to its inverse limit, which is a $W$-module, and then forms a tensor of it with $K$. The limit $\varliminf_{K}$ takes $\mathcal{A}_{\mathbf{Q}}(k)$ to $\mathbf{A}(K)$. We use the same notation for the functor induced on $\Delta \mathcal{C}_{W, \mathbf{Q}}(k)$.

As explained in [Nav87, pp. 13-14 and p. 23], given an object $G$ of $\Delta \mathbf{A}(K)$ there is the 'usual' way of giving to $s(G)$, the associated simple complex, a multiplication, making it into a DGA over $K$, 


\section{CRYstalline RATional HOMOTOPY THEORY}

depending on the choice of 'Eilenberg-Zilber transformations'. We will use the notation $s(G)$ for this DGA, whether or not we are remembering its multiplicative structure. This multiplication will not be graded-commutative in general. On the other hand, the multiplication induced on $H(s(G))$ is commutative, and it can be considered as an object of $\mathbf{A}(K)$.

To construct the Thom-Whitney algebra requires a choice of an algebraic model for differential forms on the standard simplices. Let $E_{n}$ be the algebra of global differential forms relative to $\operatorname{Spec}(K)$ on the variety $\Delta_{n}:=\operatorname{Spec}\left(K\left[t_{0}, \ldots, t_{n}\right] /\left(\sum t_{i}-1\right)\right)$. The $\Delta_{n}$ form a cosimplicial scheme in the usual manner via coface maps $\delta^{i}: \Delta_{n} \rightarrow \Delta_{n+1}$ given by

$$
\left(t_{0}, \ldots, t_{n}\right) \mapsto\left(t_{0}, \ldots, t_{i-1}, 0, t_{i}, \ldots t_{n}\right)
$$

and codegeneracy maps $s_{i}: \Delta_{n+1} \rightarrow \Delta_{n}$ given by

$$
\left(t_{0}, \ldots, t_{n+1}\right) \mapsto\left(t_{0}, \ldots, t_{i}+t_{i+1}, \ldots t_{n+1}\right),
$$

so that the $E_{n}$ form a simplicial CDGA over $K$ that we will denote by $E$. A standard computation shows that $s(E)$ only has cohomology in degree 0 of dimension 1.

Now, the Thom-Whitney algebra $s_{\mathrm{TW}}(G)$ of $G$ is by definition the simple CDGA associated to the end [Mac98] of $E \bigotimes_{K} G$ considered as a functor from $\Delta_{\text {mon }}^{\text {op }} \times \Delta_{\text {mon }}$ to commutative differential bi-graded algebras over $K$. Here, $\Delta_{\text {mon }}$ refers to the subcategory of $\Delta$ where the morphisms are strictly increasing maps. Thus, it is an object of $\mathbf{A}(K)$. We elaborate a bit on this definition (using scripts): Elements of $E \otimes G$ will be of the form

$$
\left(\sum e_{n}^{p} \otimes g^{m, q}\right)_{n, m}
$$

where $n$ is the simplicial degree, $m$ is the cosimplicial degree, and $p, q$ are the complex degrees. The elements of the end are compatible sequences (indexed by $n \in \mathbb{N}$ )

$$
\left[\sum e_{n}^{p} \otimes g^{n, q}\right]_{n}
$$

where compatibility refers to the equality:

$$
\partial_{i} \otimes 1\left[\sum e_{n+1}^{p} \otimes g^{n+1, q}\right]=1 \otimes \delta^{i}\left[\sum e_{n}^{p} \otimes g^{n, q}\right] \in E_{n} \otimes G^{n+1}
$$

for all $n$. Since we still have the complex degrees $p$ and $q$ left, the result is a commutative differential (CD) bi-graded algebra. One then takes the associated simple complex to get the CDGA $s_{\mathrm{TW}}(G)$.

Readers unfamiliar with the notion of ends should apply it to the bifunctor $E$. $\otimes S$ as an exercise, where $S$ is the cosimplicial algebra (not the complex) of singular cochains with $K$-values on a topological space $X$. (One gets Sullivan's polynomial differential forms on $X$ with values in $K$.)

We note that both $s$ and $s_{\mathrm{TW}}$ define functors $s, s_{\mathrm{TW}}: \Delta \mathbf{A}(K) \rightarrow \mathbf{C}(K)$. By using an integral version of the simplices, we could define $s_{\text {TW }}$ also for cosimplicial $W$-algebras so that the construction commutes with $\varliminf_{K}$. On the other hand, the comparison with $s$, to be discussed below, will not work at the integral level.

Given choices of geometric points over all the points of $Y$, we can construct cosimplicial Godement resolutions in the usual fashion level by level [God58] for a pro-sheaf. So from an object $C \in \mathcal{C}_{W, \mathbf{Q}}(Y)$ we obtain an object of $\Delta \mathcal{C}_{W, \mathbf{Q}}(Y)$, which we will call the Godement resolution of $C$ and denote by $G(C)$. If $A \in \mathcal{A}_{\mathbf{Q}}$, then $G(A) \in \Delta \mathcal{A}_{\mathbf{Q}}$. Applying the global section functor $\Gamma$ and the associated simple complex functor $s$, we get an object $s(\Gamma(G(C)))$ in $\mathcal{C}_{W, \mathbf{Q}}(k)$ which represents $R \Gamma(C)$. When we write $R \Gamma(C)$, therefore, we will mean this explicit pro-complex. More important for our purposes is

$$
R \Gamma_{K}(C):=\lim _{K} s(\Gamma(G(C)))=s\left({\underset{\lim }{K}}_{K}(\Gamma(G(C)))\right)
$$

It is a standard fact that if $C$ is quasi-isomorphic to $C^{\prime}$, then $R \Gamma_{K}(C)$ is $\mathrm{QI}$ to $R \Gamma_{K}\left(C^{\prime}\right)$. 


\section{KIM AND R. M. HAIN}

As mentioned above, if we start with $A \in \mathcal{A}_{\mathbf{Q}}(Y), R \Gamma_{K}(A)$ will only be a DGA over $K$, not necessarily commutative. On the other hand, we can construct the object

$$
T W(A):=s_{\mathrm{TW}}\left({\underset{\lim }{K}}^{\lim } \Gamma(G(A))\right),
$$

which lies in $\mathbf{A}(K)$.

The proofs of the following are in Navarro-Aznar's paper [Nav87].

Lemma 1 [Nav87, Theorem 2.14]. There is a natural transformation of functors on $\Delta \mathcal{A}_{\mathbf{Q}}(k)$,

$$
I: s_{\mathrm{TW}} \circ \lim _{K} \rightarrow s \circ \underset{K}{\lim _{K}}
$$

which induces quasi-isomorphisms when evaluated on objects of $\Delta \mathcal{A}_{\mathbf{Q}}(k)$, and furthermore induces an isomorphism of CDGAs at the level of cohomology.

Corollary 3 [Nav87, (4.4)]. Given $A \in \mathcal{A}_{\mathbf{Q}}(Y)$, there is a $Q I$ of complexes

$$
T W(A) \simeq R \Gamma_{K}(A)
$$

which induces an algebra isomorphism on cohomology.

Corollary 4 [Nav87, (4.6)]. If $A$ is $Q I$ to $A^{\prime}$ in $\mathcal{A}_{\mathbf{Q}}(Y)$, then $T W(A)$ is $Q I$ to $T W\left(A^{\prime}\right)$ as $C D G A s$ over $K$.

Lemma 2 [Nav87, (3.7)]. Let $C^{+}$be an object of $\Delta^{+} \mathcal{A}_{\mathbf{Q}}(k)$ with $C^{+,-1}=A$ and $C:=C^{+} \mid \Delta$. Thus we have two maps of complexes

$$
\lim _{K} A \rightarrow s_{\mathrm{TW}} \underset{K}{\lim _{K}}(C), \quad \lim _{K} A \rightarrow s \varliminf_{K}^{\lim _{K}}(C) .
$$

The natural transformation $I$ gives rise to the following commutative diagram.

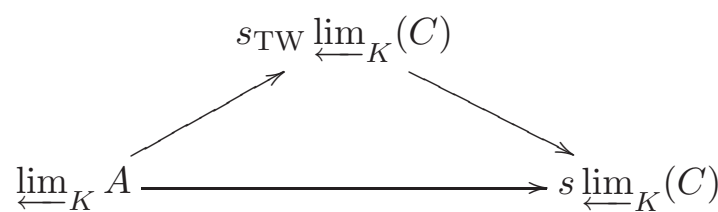

Thus, if the second map is a QI, then the first is a multiplicative quasi-isomorphism.

It will be convenient to have the Thom-Whitney functors also defined for cosimplicial sheaves of CDGAs. This is easily achieved by applying $s_{\mathrm{TW}}$ twice: If $A$ is a cosimplicial CDGA on $Y$, then each $T W\left(A^{n}\right)$ is a CDGA over $K$ and they fit together to form a cosimplicial CDGA over $K$. Applying $s_{\mathrm{TW}}$ to this gives us a CDGA that we will denote by $T W\left(A^{\cdot}\right)$. The following is easily deduced by integrating twice.

Lemma 3. We have that

$$
T W\left(A^{\cdot}\right) \simeq R \Gamma_{K}\left(s\left(A^{\cdot}\right)\right) .
$$

Similarly, suppose $Y$. is a simplicial scheme and $A$. is a CDGA over $Y$. We see then that the $T W\left(A_{n}\right)$ are objects of $\mathbf{A}(K)$ and they come together to form an object of $\Delta \mathbf{A}(K)$. We simply apply $s_{\text {TW }}$ again to get

$$
T W\left(A_{.}\right):=s_{\mathrm{TW}}\left(\left\{T W\left(A_{n}\right)\right\}_{n}\right) .
$$

The usual $R \Gamma_{K}$ on such an object can be constructed as $s\left(\left\{R \Gamma_{K}\left(A_{n}\right)\right\}_{n}\right)$ so applying the integration functor twice gives us a QI. 


\section{CRYstalline RATional HOMOTOPY THEORY}

LEMma 4. We have that

$$
T W(A .) \simeq R \Gamma_{K}\left(A_{\text {. }}\right)
$$

Corollary 5. Let $p: Y$. $\rightarrow Y$ be a simplicial hypercovering which satisfies cohomological 1-descent for the étale topology. Let $A$ be a CDGA on $Y, A$. a CDGA on $Y$, and suppose we have a $Q I$ $p^{*} A \simeq A$. Then this induces a $Q I$

$$
T W(A) \simeq T W(A .)
$$

There is a version of the functor $T W$ for filtered CDGAs [Nav87, $\S 6]$.

Denote by $A F_{\mathbf{Q}}(Y)$ the category of filtered pro-CDGAs on $Y$ up to isogeny. So an object is a pair $(A, F)$ where $A$ is an object of $\mathcal{A}_{\mathbf{Q}}(Y)$ and $F$ is a multiplicative decreasing filtration of $A$. Thus, we are given subobjects $F_{n}^{i} A_{n}$ for each $i \in \mathbb{Z}$ and each level $n$ such that $F_{n}^{i+1} A_{n} \subset F_{n}^{i} A_{n}$ and the transition maps send $F_{n}^{i} A_{n}$ surjectively to $F_{n-1}^{i} A_{n-1}$. Furthermore, we have $F_{n}^{i} F_{n}^{j} \subset F_{n}^{i+j}$ at each level. Finally, the morphisms are defined by

$$
\operatorname{Hom}_{A F_{\mathbf{Q}}(Y)}\left((A, F),\left(A^{\prime}, F^{\prime}\right)\right):=\left[\varliminf_{m}^{\lim } \underset{n}{\lim } \operatorname{Hom}\left(\left(A_{n}, F_{n}\right),\left(A_{m}^{\prime}, F_{m}^{\prime}\right)\right)\right] \otimes \mathbf{Q} .
$$

Let $(A, F)$ be an object of $\Delta A F_{\mathbf{Q}}(Y)$. Then we can define the filtered CDGA $T W(A, F)$ over $K$. in a manner entirely analogous to the previous discussion: One takes the simple filtered CDGA associated to the end of $E \otimes \varliminf_{K} \Gamma(G(A, F))$ where the filtration on the tensor product is induced by the given filtration $F$ on $\overleftarrow{A}$ and the trivial decreasing filtration $\epsilon$ of $E$ defined by

$$
\epsilon^{i}(E)= \begin{cases}E, & i \leqslant 0 \\ 0, & i>0\end{cases}
$$

We also have the functor $s$ which associates to $(A, F)$ the filtered DGA $(s(K), s(F))$. When applied to objects of $\Delta A F_{\mathbf{Q}}(k)$, we can again compose with inverse limits to end up with cosimplicial filtered CDGAs over $K$.

Lemma 5 [Nav87, Lemma 6.3, (6.7)]. The natural transformation

$$
I: s_{\mathrm{TW}} \circ \lim _{K} \rightarrow s \circ \underset{K}{\lim _{K}}
$$

of functors on $\triangle A F_{\mathbf{Q}}(k)$ discussed previously also gives a filtered quasi-isomorphism which induces in cohomology an isomorphism of filtered CDGAs.

If we denote by $R \Gamma_{K}(A, F)$ the filtered DGA

$$
\left(R \Gamma_{K}(A), s\left({\underset{\leftarrow}{K}}_{\lim } \Gamma(G(F))\right)\right)
$$

Then we have the following corollaries.

Corollary 6. There is a filtered QI

$$
T W(A, F) \simeq \lim _{\longleftarrow} R \Gamma_{K}(A, F)
$$

of complexes of $K$-vector spaces which induces an isomorphism of filtered CDGAs in cohomology.

Corollary 7. TW takes filtered QIs to filtered QIs [Nav87, (6.14)].

We now give a brief discussion of the bar complex. 


\section{KIM AND R. M. HAIN}

Let $A \in \mathbf{A}(K)$ and let $a: A \rightarrow K$ be an augmentation. Assume throughout this discussion that $A$ has connected cohomology, that is, $H^{0}(A)=K$, and has no cohomology in negative degrees. Define the bar complex $B(A, a) \in \mathbf{C}(K)$ by the formulas in [Hai87a, pp. 275-276]: Let $I$ be the augmentation ideal and

$$
B^{-s, t}(A, a)=\left(\bigotimes^{s} I\right)^{t}
$$

where the outer superscript $t$ denotes the subset of elements of degree $t$. We denote the element

$$
a_{1} \otimes \cdots \otimes a_{s}
$$

of $B^{-s, t}(A, a)$ by $\left[a_{1}|\cdots| a_{s}\right]$.

There are two differentials $d_{C}$ and $d_{I}$ (the 'combinatorial' and 'internal' differentials),

$$
d_{C}: B^{-s, t} \rightarrow B^{-s+1, t}, \quad d_{I}: B^{-s, t} \rightarrow B^{-s, t+1},
$$

given by the formulas:

$$
d_{C}\left(\left[a_{1}|\cdots| a_{s}\right]\right):=\sum_{i=1}^{s-1}(-1)^{i+1}\left[J a_{1}|\cdots| J a_{i-1}\left|J a_{i} \wedge a_{i+1}\right| a_{i+2}|\cdots| a_{s}\right]
$$

where $J(v)=(-1)^{\operatorname{deg} v} v$ and

$$
d_{I}\left(\left[a_{1}|\cdots| a_{s}\right]\right):=\sum_{i=1}^{s}(-1)^{i}\left[J a_{1}|\cdots| J a_{i-1}\left|d a_{i}\right| a_{i+1}\left|a_{i+2}\right| \cdots \mid a_{s}\right] .
$$

These differentials make the direct sum of the $B^{-s, t}$ into a double complex and we denote by $B(A, a)$ the associated total complex.

There is a filtration $\mathcal{B}$ on $B$ given by

$$
\mathcal{B}^{-s}:=\bigoplus_{u \leqslant s} B^{-u, t}
$$

called the bar filtration.

Denote by

$$
\mathcal{B}_{0} H(B(A, a)) \subset \mathcal{B}_{1} H(B(A, a)) \subset \mathcal{B}_{2} H(B(A, a)) \subset \cdots
$$

the filtration induced on the cohomology, where one notes that the signs of the indices have been reversed with respect to the bar filtration. That is,

$$
\mathcal{B}_{r} H:=\operatorname{Im}\left(H\left(\mathcal{B}^{-r}\right)\right) \text {. }
$$

Let $E_{r}$ be the spectral sequence, the Eilenberg-Moore spectral sequence, associated with the bar filtration and converging to the cohomology of the bar complex. We have for the $E_{1}$-term,

$$
E_{1}^{-s, t} \simeq B^{-s, t}(H(A)),
$$

the terms of the bar complex on the cohomology of $A$ regarded as an augmented algebra.

In particular,

$$
E_{1}^{-s, s}=\bigotimes^{s} H^{1}(A)
$$

In any case, we see that if $A$ has coherent cohomology, that is, if all the $H^{i}$ are finite-dimensional $K$-vector spaces, then the $\mathcal{B}_{r} H^{n}(B(A, a))$ are also finite-dimensional for all $n$. We note that for coherence of $\mathcal{B}_{r} H^{0}(B(A, a))$ we just need the coherence of $H^{1}(A)$. If $A$ has coherent cohomology which furthermore occurs only in bounded degrees, we also see that $\mathcal{B}_{r} H(B(A, a))$ is finite-dimensional over $K$ for each $r$. 


\section{CRYstalline RATional HOMOTOPY THEORY}

There is a multiplicative structure on $B(A, a)$ given by the formula [Hai87a, p. 278]

$$
\left[a_{1}|\cdots| a_{r}\right]\left[a_{r+1}|\cdots| a_{r+s}\right]=\sum \epsilon(\sigma)\left[a_{\sigma(1)}|\cdots| a_{\sigma(r+s)}\right],
$$

where $\sigma$ runs over all shuffles of type $(r, s)$ and $\epsilon: \Sigma_{r+s} \rightarrow\{ \pm 1\}$ is the representation of the symmetric group obtained by giving $a_{j}$ weight $-1+\operatorname{deg} a_{j}$. That is, transpositions contribute a minus sign only when switching two elements of even degree.

We also have the comultiplication:

$$
\left[a_{1}|\cdots| a_{s}\right] \mapsto \sum_{i=1}^{s}\left[a_{1}|\cdots| a_{i}\right] \otimes\left[a_{i+1}|\cdots| a_{s}\right]
$$

that combines with the multiplication to give $B(A, a)$ the structure of a differential graded Hopf algebra over $K$. This induces a commutative Hopf algebra structure on $H^{0}(B(A, a))$. The bar filtration on $B(A, a)$ is preserved by the comultiplication and hence we get a filtration of $B(A, a)$ by sub-Hopf algebras $B_{r}(A, a)$, defined to be the subalgebra generated by the $r$ th level of the bar filtration. We will be mostly interested in the induced filtration of $H(B(A, a))$, which we will denote by $H_{r}(B(A, a))$. If $H(A)$ is coherent with bounded degree and $H^{0}(A)=K$, we see that this induces a filtration of $H(B(A, a))$ by finitely generated subalgebras. Similarly, if $H^{1}$ is finite-dimensional, then $H^{0}(B(A, a))$ is filtered by finitely generated subalgebras $H_{r}^{0}(B(A, a))$.

We will need an algebraic fact about the bar complex to compare fundamental groups to homology. The following result is of course well known (cf. e.g. [Sul77]), but we were unable to locate a proof involving the maps that we need for our purposes. Therefore, we include one here.

As mentioned, with our assumptions, $H^{0}(B(A, a))$ has the structure of a non-negatively graded, commutative Hopf algebra. Each closed element $f \in A^{1}$ determines a closed element $[f]$ of $H^{0}(B(A, a))$. Since $[d f]=d[f]$ for all $f$ in the augmentation ideal, $[f]$ depends only on the class of $f$ in $H^{1}(A)$. There is therefore a well-defined linear mapping

$$
\phi: H^{1}(A)[1] \rightarrow H^{0}(B(A, a)) .
$$

Denote the bicommutative Hopf algebra generated by the vector space $V$ by $S V$. Since $H^{0}(B(A, a))$ is commutative, $\phi$ induces an algebra homomorphism

$$
\hat{\phi}: S\left(H^{1}(A)[1]\right) \rightarrow H^{0}(B(A, a)) .
$$

Since each $[f]$ is a primitive element of $H^{0}(B(A, a)), \hat{\phi}$ is a Hopf algebra homomorphism.

Proposition 1. The homomorphism

$$
\hat{\phi}: S\left(H^{1}(A)[1]\right) \rightarrow H^{0}(B(A, a))
$$

is the inclusion of the unique maximal cocommutative Hopf subalgebra.

Proof. First note that $S\left(H^{1}(A)[1]\right)$ is naturally graded by the symmetric powers of its primitives:

$$
S\left(H^{1}(A)[1]\right)=\bigoplus_{s \geqslant 0} S^{s}\left(H^{1}(A)[1]\right) .
$$

If we set

$$
B_{s} S\left(H^{1}(A)[1]\right)=\bigoplus_{t \leqslant s} S^{t}\left(H^{1}(A)[1]\right)
$$

then $S\left(H^{1}(A)[1]\right)$ is isomorphic to its associated graded object and also the homomorphism $\hat{\phi}$ is filtration preserving. One therefore has a homomorphism

$$
G r^{B} \hat{\phi}: S\left(H^{1}(A)[1]\right) \rightarrow G r^{B} H^{0}(B(A, a))=\bigoplus_{s \geqslant 0} E_{\infty}^{-s, s}
$$




\section{KIM AND R. M. HAIN}

When $1 \leqslant r<r^{\prime}<\infty$, we have inclusions

$$
\bigoplus_{s \geqslant 0} E_{r}^{-s, s} \supseteq \bigoplus_{s \geqslant 0} E_{r^{\prime}}^{-s, s} \supseteq \bigoplus_{s \geqslant 0} E_{\infty}^{-s, s}
$$

Define $\hat{\phi}_{r}$ to be the composite

$$
S\left(H^{1}(A)[1]\right) \stackrel{G r \hat{\phi}}{\longrightarrow} \bigoplus_{s \geqslant 0} E_{\infty}^{-s, s} \hookrightarrow \bigoplus_{s \geqslant 0} E_{r}^{-s, s} .
$$

Since each $E_{r}$ is a connected, graded Hopf algebra, and since $\hat{\phi}_{r}$ is injective on primitives, each $\hat{\phi}_{r}, 1 \leqslant r \leqslant \infty$, is injective. Since $G r^{B} \hat{\phi}$ is injective, $\hat{\phi}$ is also injective.

It remains to prove maximality. First, the dual of

$$
\oplus E_{1}^{-s, s}
$$

is the tensor algebra on the dual of $H^{1}(A)[1]$; its Hopf algebra structure is characterized by the fact that the dual of $H^{1}(A)[1]$ is primitive. This has, as maximal commutative quotient, the free bicommutative Hopf algebra generated by the dual of $H^{1}(A)[1]$. It follows that $\hat{\phi}_{1}$ is the inclusion of the unique maximal bicommutative sub-Hopf algebra of $\oplus E_{1}^{-s, s}$. But since

$$
\oplus E_{1}^{-s, s} \supseteq \oplus E_{2}^{-s, s} \supseteq \oplus E_{3}^{-s, s} \supseteq \cdots \supset E_{\infty}^{-s, s},
$$

it follows that $\hat{\phi}_{r}$ is the inclusion of the unique maximal bicommutative Hopf subalgebra of $\oplus E_{r}^{-s, s}$ whenever $1 \leqslant r \leqslant \infty$.

The result now follows as $\hat{\phi}_{\infty}=G r^{B} \hat{\phi}$ and since

$$
H^{0}(B(A, a))=\bigcup_{s \geqslant 0} B_{s} H^{0}(B(A, a)) .
$$

\section{Review of the De Rham-Witt complex}

We will work in the setting of log schemes, and make extensive use of the theory developed by Kato [Kat89], Hyodo [Hyo91, Hyo88], and Hyodo-Kato [HK94]. The reader should consult these articles for precise notions and notation. Furthermore, a number of important gaps in the literature have been filled in the recent preprint of Nakkajima [Nak02]. We will cite the necessary results as we proceed.

Denote by $S_{0}$ the scheme $\operatorname{Spec}(k)$ endowed with a fine log structure $L$. Unless the context makes it necessary to be careful with the distinction, we will denote by the same letter the scheme without the $\log$ structure. $L$ determines a canonical log structure on $W=W(k)$ induced by the pre-log structure that composes $L \rightarrow k$ with the Teichmüller lift. One has a similar construction for any log scheme $Y$ over $k$. By $W(Y)$, we denote the system of log schemes (or ind-log scheme) with underlying space the same as the space of $Y$, but with structure sheaves $W_{n}\left(\mathcal{O}_{Y}\right)$ and the $\log$ structures lifted with the Teichmüller character. This definition extends naturally to simplicial $\log$ schemes $Y$. over $k$ to give simplicial ind-log schemes $W(Y$.).

Denote by $S$ the scheme $\operatorname{Spec}(W)$, again with and without the $\log$ structure. Let $Y$ be a smooth, fine $\log$ scheme over $S_{0}$ of Cartier type. For each $n \geqslant 1$, Hyodo and Kato define a level $n$ De RhamWitt complex $W_{n} \omega_{Y}$ (with respect to $S_{n}$ ) which is a (sheaf of) commutative differential graded algebra(s) on the small étale site of (the underlying scheme of) $Y$ equipped with projections $\pi_{n}$ : $W_{n} \omega \rightarrow W_{n-1} \omega$. The log-De Rham complex $\Omega_{Y / S_{0}}$ occurs at the bottom level (that is, $W_{1} \omega_{Y}$ ), and in degree zero, we have $W_{n} \omega_{Y}^{0}=W_{n} \mathcal{O}_{Y}$, Serre's sheaf of Witt vectors. There are also operators,

$$
F: W_{n+1} \omega^{q} \rightarrow W_{n} \omega^{q}, \quad V: W_{n} \omega^{q} \rightarrow W_{n+1} \omega^{q},
$$




\section{Crystalline Rational homotopy theory}

extending the usual Frobenius and Verschiebung on $W \mathcal{O}$ and satisfying the identities

$$
F(a b)=F(a) F(b), \quad V(F(a) b)=a V(b)
$$

and

which implies

$$
F V=V F=p, \quad F d V=d
$$

$$
d F=p F d, \quad V d=p d V
$$

Here and henceforward, $\sigma$ denotes the Frobenius map of $W$ or $W_{n}$ for all $n$.

We denote by $W \omega_{Y}$ the object of $\mathcal{A}_{\mathbf{Q}}(Y)$, therefore, a pro-sheaf of CDGAs, given by the system of $W_{n} \omega_{Y}$. It should also be noted that $F$ is multiplicative and agrees with the usual Frobenius on $W_{n} \mathcal{O}_{Y}$. The Frobenius of $Y$ itself induces a map $\Phi$ of $W \omega_{Y}$ which is $p^{i} F$ in degree $i$.

For the purposes of defining the weight filtration, it will be useful to recall the various different constructions of the DRW complex.

One definition of $W_{n} \omega_{Y}^{i}$ is as

$$
R^{i} u_{Y / S_{n}, *}\left(\mathcal{O}_{Y / S_{n}, \text { crys }}\right)
$$

where $u_{Y / S_{n}}$ is the map from the crystalline site of $Y$ with respect to the base $S_{n}$ to the étale site of $Y$ and $\mathcal{O}_{Y / S_{n} \text {,crys }}$ is the crystalline structure sheaf. That is, $W_{n} \omega_{Y}^{i}$ is the $i$ th crystalline cohomology sheaf. Note here that the cohomology is taken with respect to the $\log$ structure on $S_{n}$ induced by $L$.

To 'compute' this sheaf, one chooses an embedding system $(Y, Z$.) for $Y / S$, that is, a simplicial $\log$ scheme $p: Y . \rightarrow Y$ which is a proper hypercovering for the étale topology together with a closed embedding $Y \hookrightarrow Z$., where $Z$. is a simplicial log scheme smooth over $S$. Let $D$. be the divided power (PD) envelope of $Y$. in $Z$. and let $\Omega_{D}$. be the associated De Rham complex. That is, $\Omega_{D_{n}}=\Omega_{Z_{n} / S} \bigotimes_{\mathcal{O}_{Z}} \mathcal{O}_{D_{n}}$ viewed as a pro-sheaf on $Y_{n}$, and these come together to form a simplicial pro-sheaf on $Y$. denoted $C_{Y / S}=C_{Y / S}(Y, Z$. ) and called the crystalline complex for this embedding system. Note that $C_{Y / S}$ is actually a simplicial pro-sheaf of CDGAs. We will denote by $p$ the map from $Y$. to $Y$ viewed as a structure map for an augmented simplicial scheme and use the same letter for the maps from the individual components of $Y . R p_{*}\left(C_{Y / S}\right)$ is then a cosimplicial object in the derived category of $\mathcal{C}_{W, \mathbf{Q}}(Y)$. If we use the natural notation $s\left(R p_{*}\left(C_{Y / S}\right)\right)$ for the complex associated to this cosimplicial object, then according to [HK94, Proposition 2.20],

$$
R u_{Y / S, *}\left(\mathcal{O}_{Y / S, \mathrm{crys}}\right) \simeq s\left(R p_{*}\left(C_{Y / S}\right)\right)
$$

canonically as pro-complexes. So

$$
W \omega_{Y}^{i} \simeq \underline{H}^{i}\left(s\left(R p_{*}\left(C_{Y / S}\right)\right)\right) .
$$

To see in this description the independence of the embedding system, one merely notes that any two embedding systems $\left(Y_{.}, Z_{.}\right)$and $\left(Y_{.}^{\prime}, Z_{.}^{\prime}\right)$ are dominated by a third $\left(Y_{.}^{\prime \prime}, Z_{.}^{\prime \prime}\right)$, which induces quasi-isomorphisms

$$
C_{Y / S}\left(Y_{.}, Z\right) \rightarrow C_{Y / S}\left(Y^{\prime \prime}, Z_{.}^{\prime \prime}\right) \leftarrow C_{Y / S}\left(Y^{\prime}, Z^{\prime}\right) .
$$

It is worth noting that the DRW complex is a local object, and that, locally, the embedding system can just be taken as $Y \hookrightarrow Z$, a lifting of $Y$ to a smooth $\log$ scheme over $S$ (which exists by [Kat89, Proposition 3.14]). Then we get the simpler formula

$$
W \omega_{Y}^{i} \simeq \underline{H}^{i}\left(\Omega_{Z}\right)
$$

These formulas just follow from the definitions, but a harder theorem [HK94, Theorem 4.19] says that

$$
p^{*}\left(W \omega_{Y}\right) \simeq C_{Y / S}
$$




\section{KIM AND R. M. HAIN}

as pro-sheaves of CDGAs on $Y$. if we take an embedding system which admits a map $W(Y$. $\rightarrow Z$. (for example, if $Z$. admits a Frobenius lift).

This is [HK94, Theorem 4.19] except that reference does not mention the multiplicative structure. However, the compatibility of this isomorphism with the multiplicative structure is contained in the proof. More precisely, the map from $C_{Y / S}$ to $p^{*}\left(W \omega_{Y}\right)$ is determined by pulling back differentials via the map $W(Y$. $) \rightarrow Z$. to the quotient of $\Omega_{W(Y) / S}$ by the differential graded ideal generated by elements of the form $d\left(a^{[i]}\right)-a^{[i-1]} d a$, where $a \in \operatorname{Ker}\left(W_{n}\left(\mathcal{O}_{Y_{\text {. }}}\right) \rightarrow \mathcal{O}_{Y}\right)$, and then mapping this quotient algebra to $p^{*}\left(W \omega_{Y}\right)$ by another algebra map. This composed map is proved to be a quasi-isomorphism.

So we get

$$
T W\left(W \omega_{Y}\right) \simeq T W\left(C_{Y / S}\right)
$$

which is a QI of CDGAs over $K$.

Henceforward, assume that $\operatorname{Spec}(k)$ is equipped with the log structure of the punctured point, determined by the map $\mathbb{N} \rightarrow k$ of monoids that sends 1 to 0 .

In the construction of the monodromy operator on the crystalline cohomology of $Y$, a key role is played by the exact sequence:

$$
0 \rightarrow W \omega_{Y}[-1] \rightarrow W \tilde{\omega}_{Y} \rightarrow W \omega_{Y} \rightarrow 0 .
$$

It is constructed as follows:

Equip Spec $W[t]$ with the $\log$ structure that takes $1 \rightarrow t$. Let $W\langle t\rangle$ be the PD envelope of the ideal $(t)$ in $W[t]$ equipped with the inverse image log structure. We have the maps

$$
\operatorname{Spec}(k) \hookrightarrow \operatorname{Spec}(W) \hookrightarrow \operatorname{Spec}(W\langle t\rangle),
$$

which actually are exact embeddings of log schemes. Thus, we can consider the crystalline cohomology of $Y$ with respect to $W$ or with respect to $W\langle t\rangle$. On the other hand, we have the smooth structure map Spec $W[t] \rightarrow \operatorname{Spec}(W)_{0}$ where we use the last subscript 0 to denote the fact that $W$ is being considered with the trivial $\log$ structure. Let $p:(Y, Z$. $) \rightarrow Y$ be an embedding system for $Y$ when we view it as a log scheme over Spec $W[t]$.

Thus, $Z$. can be viewed as a smooth simplicial log scheme over $\operatorname{Spec} W[t]$ or over $\operatorname{Spec}(W)_{0}$. We will abuse notation slightly and denote by the same $Z$. the base change of $Z / W[t]$ to $W\langle t\rangle$. Therefore, we get a PD pro-De Rham complex $\Omega_{\tilde{D}}$ for the PD envelope of $Y$ in the smooth log scheme $Z \rightarrow \operatorname{Spec}(W)$ ('the De Rham complex of the total space'), and the pro-De Rham complex $\Omega_{D}$ for the PD envelope of $Y$. in $Z$. $\rightarrow$ Spec $W\langle t\rangle$ ('the relative De Rham complex'), which give rise to an exact sequence of pro-sheaves on $Y$ :

$$
0 \rightarrow \Omega_{D}[-1] \rightarrow \Omega_{\tilde{D}} \rightarrow \Omega_{D} \rightarrow 0 .
$$

The first map takes the differential $\alpha$ to $\alpha \wedge d t / t$. Taking the tensor product with $W$ preserves the exactness [HK94, Lemma 2.22], giving us

$$
0 \rightarrow \Omega_{D}[-1] \bigotimes_{W\langle t\rangle} W \rightarrow \Omega_{\tilde{D}} \bigotimes_{W\langle t\rangle} W \rightarrow \Omega_{D} \bigotimes_{W\langle t\rangle} W \rightarrow 0
$$

Also, $\Omega_{D} \bigotimes_{W\langle t\rangle} W$ is a crystalline complex for $Y / S$. Now, $W \tilde{\omega}^{i}$ is by definition $R^{i} p_{*}\left(\Omega_{\tilde{D}} \bigotimes_{W\langle t\rangle} W\right)$.

We remark that $W \tilde{\omega}$ carries operators $F, V, d, \pi$ satisfying the same relations as and compatible with those of $W \omega$ : One just repeats the construction of [HK94, p. 246-249] using the $W$ flatness of $\Omega_{\tilde{D}} \otimes W$.

To elaborate a bit on the local description, assume that we have a single smooth log scheme $Z$ over $W[t]$ such that $X:=Z \bigotimes_{W[t]} W$ is a smooth $W$ lift of $Y$. Equip $W[t]$ with the Frobenius which 


\section{CRYSTALline RATIONAL HOMOTOPY THEORY}

is the usual Frobenius on $W$ and such that $t \mapsto t^{p}$. Also assume that $Z$ admits a Frobenius lift $\phi$ (compatible with the Frobenius on $W[t]$ ) and that $Z$ is a lifting of $Z \bigotimes_{W} k$ which is of Cartier type. In particular, we are assuming that $Z_{0} \hookrightarrow Z$ is an exact closed immersion of log schemes. Such liftings always exist locally. In this case, $\Omega_{Z / W}$ and $\Omega_{Z / W[t]}$ both carry operators $\bar{F}$ which by definition is $\left(1 / p^{i}\right) \phi^{*}$ on the $i$-forms ( $p^{i}$-divisibility of $\phi^{*}$ on $i$-forms follows from the exactness of the immersion). Also, the exact sequence above becomes

$$
0 \rightarrow \Omega_{X / W}[-1] \rightarrow \Omega_{Z / W} \bigotimes_{W[t]} W \rightarrow \Omega_{X / W} \rightarrow 0
$$

Here, we have used the fact that, for example,

$$
\Omega_{\tilde{D} / W}=\Omega_{Z / W} \bigotimes_{W[t]} W\langle t\rangle .
$$

We have the following important formula:

$$
d^{-1}\left(p^{n} C^{i+1}\right)=\sum_{0 \leqslant k \leqslant n} p^{k} \bar{F}^{n-k}\left(C^{i}\right)+\sum_{0 \leqslant k \leqslant n-1} \bar{F}^{k}\left(d C^{i-1}\right),
$$

where $C$ is either $\Omega_{Z / W}$ or $\Omega_{X / W}$. The proof is exactly as in [Ill79, Proposition 0.2.3.13], where we substitute the Cartier isomorphism (4.1.2) and the definition (4.3) of [HK94] for Illusie's discussion starting in 0.2.2.2 and up to Proposition 2.3.13. This was pointed out by Jannsen in commentaries (6) and (11) to Lemma 1.4 of [Hyo91].

Formula (2) implies that

$$
\underline{H}^{i}\left(\Omega_{Z / W} \bigotimes_{W[t]} W_{n}\right) \rightarrow \underline{H}^{i}\left(\Omega_{X / W_{n}}\right)
$$

is surjective for each $n$ : Let $a \in \Omega_{X / W}^{i}$ be closed $\bmod p^{n}$. Then

$$
a=\sum_{0 \leqslant k \leqslant n} p^{k} \bar{F}^{n-k}\left(c^{k}\right)+\sum_{0 \leqslant k \leqslant n-1} \bar{F}^{k}\left(d b^{k}\right)
$$

for some $c^{k}$ and $b^{k}$. Lift these to $C^{k}$ and $B^{k}$ in $\Omega_{Z / W}$. Then

$$
a^{\prime}=\sum_{0 \leqslant k \leqslant n} p^{k} \bar{F}^{n-k}\left(C^{k}\right)+\sum_{0 \leqslant k \leqslant n-1} \bar{F}^{k}\left(d B^{k}\right)
$$

is an element of $\Omega_{Z / W}$ which is a cocycle mod $p^{n}$ and maps to $a$. Now take the image of $a^{\prime}$ in $\Omega_{Z / W} \otimes W$ to get the required surjectivity. Hence, we get the exactness (1) that we want, as soon as we know that the sequence is independent of the embedding system. (It also shows that the transition map between levels is surjective.) The proof that $W \tilde{\omega}_{Y}$ and this sequence are independent of the embedding system proceeds exactly as the proof of the independence of the crystalline cohomology: Any two embedding systems can be dominated by a third, and the two sequences possess maps to the third which are isomorphisms. That is, once we know that the sequences are exact and we have isomorphisms for the maps on either end, the middle map is also an isomorphism.

It has been pointed out to us by the referee that the papers [Hyo91] and [HK94] do not deal with the question of whether the exact sequence which is defined at each level is compatible with the projection maps. This issue was remedied carefully in [Nak02, § 6]. Also, in [Nak02, § 10], the Frobenius compatibility of the exact sequence is checked.

The monodromy operator in crystalline cohomology is the coboundary map $H^{i}(W \omega) \rightarrow H^{i}(W \omega)$ arising from this exact sequence.

An alternative construction of the monodromy operator goes as follows: Define a CDGA $W \tilde{\omega}[u]$ by adjoining to $W \tilde{\omega}$ the divided powers of a variable $u$ in degree zero. That is, $W \tilde{\omega}[u]$ is the 


\section{KIM AND R. M. HAIN}

CDGA generated by $W \tilde{\omega}$ and variables $u^{[i]}, 0,1,2, \ldots$, satisfying the relations $d u^{[i]}=(d t / t) u^{[i-1]}$ and $u^{[0]}=1$. Thus, $W \tilde{\omega}[u]$ is a 'divided-power' Hirsch extension.

Consider the natural map $r: W \tilde{\omega}[u] \rightarrow W \omega$ obtained by composing the two maps $W \tilde{\omega}[u] \rightarrow$ $W \tilde{\omega} \rightarrow W \omega$.

Lemma 6. The map $r$ is a quasi-isomorphism.

Proof. Clearly $r$ is surjective. We can regard $W \tilde{\omega}[u]$ as a double complex whose squares look like

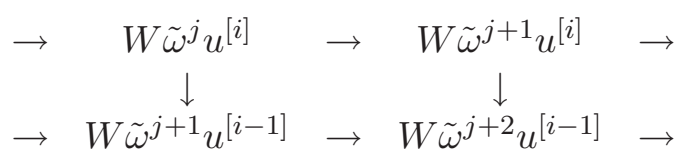

and we need to prove that the columns are exact for $i \geqslant 2$. However, the map

$$
W \tilde{\omega}^{j} u^{[i]} \rightarrow W \tilde{\omega}^{j} u^{[i-1]}
$$

takes $\alpha u^{[i]}$ to $\alpha d t / t u^{[i-1]}$ and the exact sequence (1) implies the exactness of the sequence

$$
\rightarrow W \tilde{\omega} \rightarrow W \tilde{\omega} \rightarrow W \tilde{\omega} \rightarrow
$$

where each horizontal arrow is given by wedging with $d t / t$, and the columns of our double complex come exactly from this sequence. This proves the lemma.

$W \tilde{\omega}[u]$ also carries an $F$-operator by defining $F\left(u^{[i]}\right)=p^{i} u^{[i]}$.

Now define the monodromy operator on $W \tilde{\omega}[u]$ to be the $W \tilde{\omega}$-linear map that takes $u^{[i]}$ to $u^{[i-1]}$. The quasi-isomorphism above allows us to transfer this to $W \omega$ in the derived category which hence gives us a monodromy operator in cohomology. These identities and $F(d t / t)=d t / t$ give us the relation

$$
p \Phi N=N \Phi,
$$

where $\Phi$ is the operator on $W \omega[u]$ and $W \tilde{\omega}[u]$ equal to $p^{i} F$ in degree $i$.

Lemma 7. Viewed as an endomorphism of $R \Gamma(W \omega)$ in the derived category of pro-complexes of $W$-modules, this monodromy operator agrees with that given by the coboundary map of the exact sequence (1).

Proof. To compute $N$ on a cocycle $a$ of $R \Gamma(W \omega)$, one lifts it to a cocycle $a_{0}+a_{1} u^{[1]}+\cdots \in$ $R \Gamma(W \tilde{\omega})[u]$, that is, an element satisfying $\left[a_{0}\right]=a$ and $d\left(a_{0}+a_{1} u^{[1]}+\cdots\right)=0$, applies $N$ to get $a_{1}+a_{2} u^{[1]}+\cdots$ and projects back to $R \Gamma(W \omega)$ to get $\left[a_{1}\right]$. The closedness gives us $d a_{0}=a_{1} d t / t$. Thus, $\left[a_{1}\right]$ is the second component of a cocycle $\left(a_{0},\left[a_{1}\right]\right)$ in the cone of the map $R \Gamma(W \omega[-1]) \rightarrow R \Gamma(W \tilde{\omega})$ from (1) which maps to $\left[a_{1}\right]$ via the projection of $(1)$. This proves the claim.

To get the monodromy operator on the homotopy groups, we need a slight modification of $W \tilde{\omega}$ to accommodate augmentations. For this, it is important to assume that $y \in Y$ is a point such that the $\log$ structure on $Y$ is locally of the form $f^{*} L$ near $y$, where $L$ is the log structure (of the punctured point) on $k$ and $f: Y \rightarrow \operatorname{Spec}(k)$ is the structure map. That is, we assume that $y: S_{0} \rightarrow Y$ is an exact embedding of log schemes.

Let $I$ be the kernel of the natural augmentation map of sheaves $W \omega \rightarrow y_{*} W$. Then $I$ can be resolved by an ideal in $W \tilde{\omega}[u]$ as follows: Let $Z$ be a local lifting (around $y$ ) to a smooth log scheme over $W[t]$ as above and let $X=Z \bigotimes_{W[t]} W$. Lift $y$ to a point $x$ of $X$. As above, we have the exact sequence

$$
0 \rightarrow \Omega_{D} \otimes W[-1] \rightarrow \Omega_{\tilde{D}} \otimes W \rightarrow \Omega_{D} \otimes W \rightarrow 0
$$

and we have $\Omega_{D} \otimes W \simeq \Omega_{X}$. 


\section{CRYSTALline RATIONAL HOMOTOPY THEORY}

There is a natural DG ideal $\tilde{J} \subset \Omega_{\tilde{D}} \otimes W$ defined to be the kernel of the evaluation at $x$ in degree 0 , equal to $\Omega_{\tilde{D}}^{i} \otimes W$ for $i \geqslant 2$, and in degree 1 consists of the ' 1 -forms whose coefficient of $d t / t$ vanishes at $x$ '. Only the degree-one part requires explanation: Since the $\log$ structure on $Z$ is locally of the form $g^{*}(N)$ near $x$, where $g: Z \rightarrow \operatorname{Spec}(W[t])$ is the structure map and $N$ is the $\log$ structure defined by the divisor $t=0$, an element of $\Omega_{\tilde{D}}^{1} \otimes W$ can be locally written as

$$
a d t / t+b
$$

near $x$ where $b$ is a 'usual' regular differential form near $x$. Therefore, $a(x)$ is well defined, as is the subsheaf of 1 -forms for which $a(x)$ is zero.

Also, denote by $J$ the augmentation ideal in $\Omega_{X}$ corresponding to evaluation at $x$. Then an easy local calculation gives us an exact sequence

$$
0 \rightarrow J[-1] \rightarrow \tilde{J} \rightarrow J \rightarrow 0 .
$$

The cohomology sheaf of $J$ (regarded as a pro-sheaf on $Y$ ) is naturally identified with the augmentation ideal $I \subset W \omega_{Y}$. Denote by $\tilde{I}$ the cohomology sheaf of $\tilde{J}$.

Lemma 8. We have a map of exact sequences as follows.

$$
\begin{array}{ccccccccc}
0 & \rightarrow & I[-1] & \rightarrow & \tilde{I} & \rightarrow & I & \rightarrow & 0 \\
& & \downarrow & & \downarrow & & \downarrow & & \\
0 & \rightarrow & W \omega_{Y}[-1] & \rightarrow & W \tilde{\omega}_{Y} & \rightarrow & W \omega_{Y} & \rightarrow & 0
\end{array}
$$

Proof. We need only check that the sequence

$$
0 \rightarrow I[-1] \rightarrow \tilde{I} \rightarrow I \rightarrow 0
$$

is exact. But this follows by the same argument as for the complex without augmentations.

Now, we can form the complex $\tilde{I}[u]$ by adjoining divided powers of $\log (t)$ as above, which gives us a resolution of $I$. Then $\tilde{I}[u]$ carries an operator $N$ again defined by differentiating with respect to $u$.

\section{The crystalline fundamental group}

Let $f: Y \rightarrow S_{0}$ be as in the previous section, except we further assume that $Y$ is connected and proper. Let $y \in Y$ be a $k$-rational point which is an exact embedding of log schemes.

Let $A_{Y}:=T W\left(W \omega_{Y}\right)$, which we will call the crystalline rational homotopy type of $Y$. This is a CDGA over $K$. It is equipped with a Frobenius $\Phi$ and a monodromy operator $N$ as described in the previous section. The point $y$ gives rise to an augmentation map $a_{y}: A_{Y} \rightarrow K$ as follows: There is a map $\Gamma\left(G\left(W \omega_{Y}\right)\right) \rightarrow G(W)$ induced by the map that evaluates elements of $W \mathcal{O}_{Y}$ at $y$ and sends higher-degree elements to zero. (Here, the Godement resolution $G(W)$ can be taken to be that on the Zariski site of $W$.) Note that this is the map induced by the map of sheaves

$$
W \omega \rightarrow y_{*}(W) \text {. }
$$

This induces a map $A_{Y} \rightarrow A_{k}$, where $A_{k}$ consists of compatible collections of forms on the algebraic $K$ simplices $\Delta_{n}$, or in other words $\lim \Gamma\left(\Omega_{\Delta_{n}}\right)$. Evaluating at the zero-dimensional simplex induces a QI $A_{k} \rightarrow K$, which gives us our augmentation. From the definition, we see that the augmentation ideal is QI to $T W(I)$, where $I$ denotes the kernel of the map $W \omega \rightarrow y_{*}(W)$.

We have that $B(Y, y):=B\left(A(Y), a_{y}\right)$ is a DGA over $K$ while $H(B(Y, y))$ is a $K$-CDGA. Now define the $K$-algebra $\operatorname{Cr}(Y, y)$ by the formula

$$
C r(Y, y):=H^{0}(B(Y, y)) \text {. }
$$




\section{KIM AND R. M. HAIN}

Thus, $\operatorname{Cr}(Y, y)$ is a $K$-Hopf algebra and has a natural filtration $C r_{r}(Y, y)=H_{r}^{0}(B(Y, y)), r=$ $0,1,2, \ldots$ by finitely generated subalgebras. (Recall here that $H_{r}^{0}(B(Y, y))$ is the subalgebra generated by the $r$-level of the bar filtration. This is also clearly closed under the comultiplication.)

Definition 1. The crystalline fundamental group $\pi_{1}^{\mathrm{cr}}(Y, y)$ is the pro-algebraic group defined by the inverse system

$$
\pi_{1}^{\mathrm{cr}}(Y, y)_{r}:=\operatorname{Spec}\left(C r_{r}(Y, y)\right)
$$

The $K$-points of $\pi_{1}^{\mathrm{cr}}(Y, y)_{r}$ are therefore the group-like elements in the dual Hopf algebra $C r_{r}(Y, y)^{*}$.

Now assume that $S_{0}$ has the log structure $L$ of the punctured point and that the log structure $N$ on $Y$ satisfies $N_{y}=f^{*}(L)_{y}$. Recall that we have a quasi-isomorphism from $T W(\tilde{I}[u])$ to $T W(I)$. Thus, we can also compute the crystalline $\pi_{1}$ using the bar complex on the first algebra, which we will denote by $\tilde{B}(Y, y)$. Since the monodromy operator clearly induces a derivation on $T W(\tilde{I}[u])$, we see that it extends naturally to the tensor product derivation on $\tilde{B}(Y, y)$, which commutes with the differential, and hence on $H^{0}(\tilde{B}(Y, y)) \simeq \operatorname{Cr}(Y, y)$. Thus, $N$ is naturally realized as a vector field on $\pi_{1}^{\mathrm{cr}}(Y, y)$. Directly from the formulas for the comultiplication and the fact that $N$ is extended to the bar complex via tensor products, we see that $N$ is compatible with the Hopf algebra structure, and hence induces a $K$-linear map of $\operatorname{Lie}\left(\pi_{1}^{\mathrm{cr}}(Y, y)\right)$, since this last is just the dual to the indecomposables

$$
Q C r(Y, y):=H^{0}(B(Y, y))^{+} /\left[H^{0}(B(Y, y))^{+} H^{0}(B(Y, y))^{+}\right] .
$$

Here, the superscript $(+)$ refers to the positively graded part. Again because it is defined by a tensor product, $N$ is also compatible with the bar filtration, hence induces vector fields on all of the $\pi_{1}^{\mathrm{cr}}(Y, y)_{r}$.

On $\operatorname{Cr}(Y, y)$ as well, the Frobenius and monodromy operators satisfy the relation

$$
p \Phi N=N \Phi,
$$

since this holds at the level of the CDGA $W \tilde{\omega}[u]$.

By Proposition 1 of $\S 3$, we get the relation between the crystalline fundamental group and crystalline cohomology: There is a natural isomorphism

$$
H_{1}^{\mathrm{cr}}(Y, K) \simeq \pi_{1}^{\mathrm{cr}}(Y, y)^{a b}
$$

where the left-hand side denotes the dual of $H_{\mathrm{cr}}^{1}(Y, K)$, the degree-1 crystalline cohomology with coefficients in $K$, and the right-hand side is the abelianization of the crystalline fundamental group. Since the isomorphism is induced by the inclusion

$$
I[1] \hookrightarrow B(Y, y)
$$

of the augmentation ideal of $T W\left(W \omega_{Y}\right)$ into the bar complex, it respects the actions of $\phi$ and $N$.

One can also phrase this relation in terms of the crystalline Lie algebra as

$$
H_{1}\left(\operatorname{Lie}\left(\pi_{1}^{\mathrm{cr}}(Y, y)\right)\right) \simeq H_{1}^{\mathrm{cr}}(Y)^{*},
$$

since the first homology of a Lie algebra is its abelianization.

\section{The weight filtration in the semi-stable case: proof of Theorem 1}

We give a few definitions preliminary to our discussion of the weight filtration. For this discussion, let $k$ now be a finite field with $q=p^{d}$ elements and let $M$ be an $F$-isocrystal over $k$, i.e. a vector space over $K$ equipped with a $\sigma$-linear bijective map $F: M \rightarrow M$. We say $M$ is pure of weight $i$ if 


\section{CRYstalline RATional HOMOTOPY THEORY}

$M$ is finite-dimensional, and the $K$-linear map $F^{d}$ has an integral characteristic polynomial whose roots all have absolute value $q^{i / 2}$. We say $M$ is mixed [Fal90] if it has an increasing (weight-)filtration whose associated graded objects are pure. We will denote the weight filtration by $P$ ('poids') because the letter $W$ is used for the DRW complex.

A mixed Frobenius complex is a triple $(M, N, P)$ of complexes of $F$-isocrystals $M$ and $N$, where $N$ is equipped with an increasing filtration $P$ which is degree-wise exhaustive and separated $\left(P_{i} N_{d}=\right.$ $N_{d}$ for $i \gg 0$ and $P_{i} N_{d}=0$ for $\left.i \ll 0\right)$ and a quasi-isomorphism $M \simeq N$ of $F$-isocrystals, such that for the spectral sequence associated to the filtration $P$,

$$
E_{1}^{p, q}=H^{q-p}\left(G r_{p} N\right)
$$

is a pure $F$-isocrystal of weight $q$. In particular, the spectral sequence degenerates at $E_{2}$. Morphisms between any of the previous objects are required to respect all structures: that is, the Frobenius, the filtration and the map between the two complexes $M$ and $N$.

The proof of the following is an easy consequence of the definitions and the Künneth formula.

Lemma 9. Let $\left(M_{1}, N_{1}, P_{1}\right)$ and $\left(M_{2}, N_{2}, P_{2}\right)$ be two mixed Frobenius complexes. Then

$$
\left(M_{1} \otimes M_{2}, N_{1} \otimes N_{2}, P_{1} \otimes P_{2}\right)
$$

is a mixed Frobenius complex.

Define a mixed Frobenius algebra to be a mixed Frobenius complex $(M, N, P)$ where both $M$ and $N$ are DGAs with multiplicative Frobenii, the QI $M \simeq N$ respects the algebra structure, and the filtration $P$ is multiplicative. We will also assume that all DGAs have connected cohomology.

An augmentation on $(M, N, P)$ is a map of mixed Frobenius algebras to $(K, K, t)$, where $K$ is the pure crystal of weight zero with $\sigma$ as Frobenius, and $t$ is the trivial filtration such that $t_{0}(K)=K$ and $t_{-1}(K)=0$. If $(I, J, P)$ denotes the pair of kernels of an augmentation with the induced filtration, it is clear that $I$ is still quasi-isomorphic to $J$. Also, since the filtration on $J$ is induced, we clearly have an inclusion of $E_{0}$-terms $E_{0}^{P}(J) \subset E_{0}^{P}(N)$. Now, suppose we have $[j] \in G r_{k}(J)$ such that $[j]=[d n]$ for some $n \in N$. Since $d$ is $K$-linear, we can always subtract from $n$ its image under the augmentation. Hence we get $[j]=\left[d n^{\prime}\right]$ for $n^{\prime} \in J$.

Thus, we conclude that the $E_{1}$ of $J$ is a sub-F-isocrystal of the $E_{1}$ for $N$. Therefore, $(I, J, P)$ is a mixed Frobenius complex.

Given an augmentation on the mixed Frobenius algebra $(M, N, P)$, we can construct the bar complexes $(B(M), B(N), B(P))$, where the filtration $B(P)$ is the convolution [Zuc85, A.2] of the filtration $P$ and the increasing bar filtration, that is, $B_{n}:=\mathcal{B}^{-n}$.

Lemma 10. If $(M, N, P)$ is a mixed Frobenius algebra, then $(B(M), B(N), B(P))$ is a mixed Frobenius complex. If $(M, N, P)$ is furthermore commutative, then the bar complex is a mixed Frobenius algebra with a comultiplication which is a morphism of mixed Frobenius algebras (from the given algebra to the tensor product).

Proof. Let $I$ be the augmentation ideal for $N$. We examine the spectral sequence for the filtration on $B(N)$. By the convolution formula [Zuc85, A.2], we see that

$$
G r_{n} B(N)=\bigoplus_{s+t=n} G r_{t}^{P} G r_{s}^{B} B(N)
$$




\section{KIM AND R. M. HAIN}

where $G r^{B}$ refers to the graded objects for the bar filtration. So

$$
\begin{aligned}
E_{0}^{-n, q} & =\bigoplus_{s+t=n} G r_{t}^{P} G r_{s}^{B} B(N)^{q-n} \\
& =\bigoplus_{s+t=n} G r_{t}^{P} B(N)^{-s, q+s-n} \\
& =\bigoplus_{s+t=n} G r_{t}^{P}\left(\bigotimes^{s} I\right)^{q+s-n} \\
& =\bigoplus_{s+t=n} G r_{t}^{P}\left(\bigotimes^{s} I\right)^{q-t} .
\end{aligned}
$$

Also one checks readily that the combinatorial differential is zero on $E_{0}$, so one gets

$$
E_{1}^{-n, q}=\bigoplus_{s+t=n} H^{q-t}\left(G r_{t}^{P}\left(\bigotimes^{s} I\right)\right) .
$$

Since $\bigotimes^{s} I$ is a mixed Frobenius complex, we see that this last object is a pure crystal of weight $q$ as desired.

The second sentence follows directly from the formulas for the multiplication and the comultiplication on the bar complex (that is, the fact that they preserve tensor degrees).

The weight filtration in log-crystalline cohomology was studied by Mokrane [Mok93]. The non-trivial issue of compatibility of the weight filtrations with the projection maps was proved in the paper [Nak02] of Nakkajima.

For the purposes of this section, $S_{0}=\operatorname{Spec}(k)$ is equipped with the log structure $L$ of the punctured point and we assume that $(Y, M)$ has a log structure which locally fits into a Cartesian diagram

$$
\begin{array}{ccccc}
(Y, M) & \hookrightarrow & X & \hookrightarrow & (Z, N) \\
\downarrow & & \downarrow & & \downarrow \\
S_{0} & \hookrightarrow & S & \hookrightarrow & \operatorname{Spec}\left(W[t], N_{0}\right)
\end{array}
$$

where $Z$ is a $W[t]$ scheme which is smooth over $W$ with the property that the divisor $X$ over $t=0$ is of normal crossing with special fiber $Y$ and $N$ is the $\log$ structure associated to the divisor $E=X+H$ where $H$ is a divisor which is relatively of normal crossing meeting $X$ transversally. That is, in étale coordinates,

$$
\begin{aligned}
Z & =\operatorname{Spec} W\left[t_{1}, \ldots, t_{n}\right], \\
X & =\operatorname{Spec} W\left[t_{1}, \ldots, t_{n}\right] /\left(t_{1} \cdots t_{a}\right) .
\end{aligned}
$$

Here $E$ is defined by $t_{1} t_{2} \cdots t_{b}=0$ for some $b \geqslant a$, and the map $Z \rightarrow \operatorname{Spec}(W[t])$ is given by

$$
t \rightarrow t_{1} t_{2} \cdots t_{a} .
$$

Also, $N_{0}$ is the $\log$ structure associated with the divisor $t=0$. Assume also that $Z$ has a Frobenius lift $F$ such that $F(t)=t^{p}$ (unit). Such a $(Z, N)$ is called an 'admissible lifting'.

We also assume that $Y$ itself is globally the union of smooth components $Y_{1}, \ldots, Y_{c}$ which intersect transversally.

At this point we again remind the reader of our convention: Various pro-sheaves of $W$-modules will occur in the following discussion. We will treat them as though they were ordinary sheaves unless serious confusion is likely to result. This is especially important to remember in the discussion of various cohomology sheaves, since, for example, if $C=\left\{C_{n}\right\}$ is a pro-complex, so that each $C_{n}$ is a $W_{n}$-module, then $\underline{H}(C)$ will mean the pro-system $\left\{\underline{H}\left(C_{n}\right)\right\}$. 


\section{Crystalline RAtional homotopy theORY}

Choose a local admissible lift as above. Use the notation $\tilde{\omega}:=\Omega_{Z}(\log E) \bigotimes_{\mathcal{O}_{Z}} \mathcal{O}_{X}$ and $\omega:=$ $\tilde{\omega} /(d t / t)$ so that $W \tilde{\omega}=\underline{H}(\tilde{\omega})$ and $W \omega=\underline{H}(\omega)$. Then $\Omega_{Z}(\log E)$ is equipped with the usual weight filtration $P^{\prime}$ given by the number of $\log$ poles, so that $\tilde{\omega}$ receives a weight filtration defined by

$$
P_{j}^{\prime} \tilde{\omega}=\operatorname{Im}\left(\left(P_{j}^{\prime} \Omega_{Z}(\log E)\right) \otimes \mathcal{O}_{X} \rightarrow \tilde{\omega}\right) .
$$

Therefore, $W \tilde{\omega}$ is naturally equipped with a weight filtration:

$$
P_{j}^{\prime} W \tilde{\omega}:=\operatorname{Im}\left[\underline{H}\left(P_{j}^{\prime} \tilde{\omega}\right) \rightarrow W \tilde{\omega}\right] .
$$

In fact, Mokrane proves that the map

$$
\underline{H}\left(P_{j}^{\prime} \Omega_{Z}(\log E) \otimes \mathcal{O}_{X}\right) \rightarrow W \tilde{\omega}
$$

is injective. In $[\mathrm{Nak} 02, \S 6]$, it is shown that the weight filtration defined in this way for each level is actually compatible with the projection maps, thereby showing that the filtration is well defined on the pro-complex.

Let $E^{i}$ denote the disjoint union of the $(i+1)$-fold intersections of the fiber over $\operatorname{Spec}(k)$ of the components of $E$. That is:

$$
E^{i}=\coprod_{|I|=i+1} \cap_{j \in I}\left(E_{j} \cap Y\right)
$$

In Mokrane [Mok93], where the horizontal component is not considered, we had

$$
G r_{n}^{P^{\prime}} W \tilde{\omega}=W \omega_{E^{n}[-n](-n) .}
$$

Here, $[-n]$ refers to a shift of complex degree while $(-n)$ is a Tate twist for the Frobenius action. It is important to note here that the $E^{i}$ are proper smooth varieties over $k$ and that the graded pieces are the usual DRW complexes for smooth varieties.

The Hyodo-Steenbrink complex is the simple complex associated to the double complex

$$
W A^{i j}:=W \tilde{\omega}^{i+j+1} / P_{j}^{\prime} W \tilde{\omega}^{i+j+1}, \quad i, j \geqslant 0,
$$

where the differential $d^{\prime}: W A^{i j} \rightarrow W A^{i+1, j}$ is induced by $(-1)^{j} d, d$ being the differential of $W \tilde{\omega}$, and $d^{\prime \prime}: W A^{i j} \rightarrow W A^{i, j+1}$ is multiplication by $d t / t$.

The map

$$
W \tilde{\omega} \rightarrow W A \quad \alpha \mapsto \alpha d t / t
$$

factors to a map

$$
W \omega \rightarrow W A
$$

which is proved to be a quasi-isomorphism. (The proof in Mokrane is completed in [Nak02].) Unfortunately, this complex is not a CDGA, so we cannot use it to compute the fundamental group. We also need to take a little care to incorporate the contributions of the horizontal component.

An alternative construction in the Hodge-theoretic context was given in [Hai87b], and we will use a De Rham-Witt analogue of that construction. It is based on the simplicial scheme obtained from the components of $Y$, as occurs often in mixed-Hodge theory.

Let $Y^{i}$ denote the disjoint union of the $(i+1)$-fold intersections of the components of $Y$. That is:

$$
Y^{i}=\coprod_{|I|=i+1} \cap_{j \in I} Y_{j}
$$

For the local admissible lift, we also get the components $X_{j}$ of $X$ and the corresponding $(i+1)$-fold intersections $X^{i}$. We also use the obvious notations $Y_{I}$ and $X_{I}$ for intersections of components indexed by $I$. 


\section{KIM AND R. M. HAIN}

Regard the $\mathcal{O}_{X^{i}}$ as forming a cosimplicial pro-sheaf on $Y$.

LEMma 11. The associated simple complex

$$
\mathcal{O}_{X^{0}} \rightarrow \mathcal{O}_{X^{1}} \rightarrow \cdots
$$

gives a resolution of $\mathcal{O}_{X}$.

Proof. By localizing in the étale topology, we may assume that the sequence with $\mathcal{O}_{X}$ appended at the left end is given by

$$
W\left[t_{1}, \ldots, t_{n}\right] /\left(t_{1} \cdots t_{a}\right) \rightarrow \prod_{i} W\left[t_{1}, \ldots, t_{n}\right] /\left(t_{i}\right) \rightarrow \prod_{i<j} W\left[t_{1}, \ldots, t_{n}\right] /\left(t_{i}, t_{j}\right) \rightarrow \cdots
$$

We have canonical isomorphisms

$$
W\left[t_{1}, \ldots, t_{n}\right] /\left(t_{i_{1}}, \ldots, t_{i_{k}}\right) \simeq W\left[t_{1}, \ldots, \widehat{t_{i_{1}}}, \ldots, \widehat{t_{i_{k}}}, \ldots, t_{n}\right],
$$

where the $\hat{\imath}$ indicates omission of a variable. Therefore, the complex

$$
W\left[t_{1}, \ldots, t_{n}\right] \rightarrow \prod_{i} W\left[t_{1}, \ldots, t_{n}\right] /\left(t_{i}\right) \rightarrow \prod_{i<j} W\left[t_{1}, \ldots, t_{n}\right] /\left(t_{i}, t_{j}\right) \rightarrow \cdots
$$

becomes isomorphic to the tensor product (over $W$ ) of the complexes

$$
W\left[t_{i}\right] \rightarrow W\left[t_{i}\right] /\left(t_{i}\right)
$$

for $i=1, \ldots, a$ and the single term complex $W\left[t_{a+1}, \ldots, t_{n}\right]$. Therefore, the Künneth formula tells us that the only cohomology for this tensor product complex occurs at the leftmost end, and is the image of the map $\left(t_{1}\right) \otimes\left(t_{2}\right) \otimes \cdots \otimes\left(t_{a}\right)$ in $W\left[t_{1}, t_{2}, \ldots, t_{n}\right]$, i.e. the ideal $\left(t_{1} \cdots t_{a}\right)$. This finishes the proof of the lemma.

Corollary 8. Let $J_{i}$ be the ideal defining $X_{i}$ and $J=J_{1} \cdots J_{a}$ be the ideal defining $X$. Then we have an exact sequence of sheaves in the étale topology:

$$
J_{1} J_{2} \cdots J_{a} \rightarrow \oplus J_{i} \rightarrow \bigoplus_{i<j}\left(J_{i}+J_{j}\right) \rightarrow \cdots
$$

Proof. Consider the following diagram:

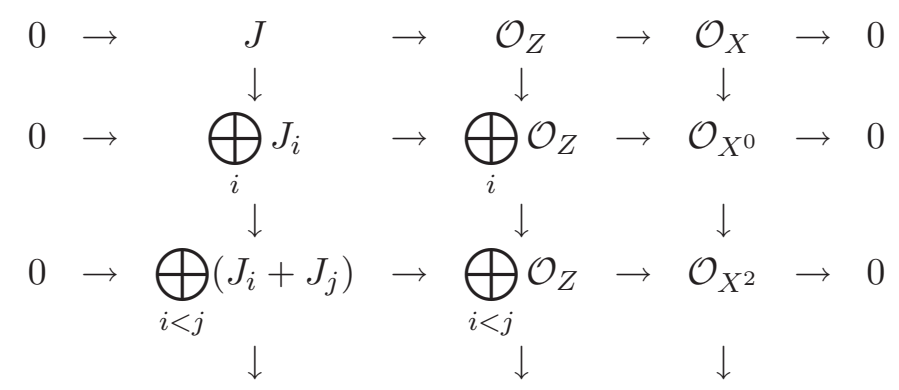

All the rows are exact. The last column is exact by the previous lemma. The middle column is exact for combinatorial reasons: The $i$ th level down is $\mathcal{O}_{Z}$ tensored with $i$ copies of the acyclic complex $\mathbb{Z} \rightarrow \mathbb{Z}$. Therefore, the first column is exact.

Since $\Omega_{Z}(\log E)$ is flat over $\mathcal{O}_{Z}$, we see that the pro-sheaves

$$
\tilde{\omega}_{X^{i}}=\tilde{\omega}_{X} \otimes \mathcal{O}_{X^{i}}=\Omega_{Z}(\log E) \bigotimes_{\mathcal{O}_{Z}} \mathcal{O}_{X^{i}}
$$




\section{Crystalline Rational homotopy theory}

also give a cosimplicial resolution of $\tilde{\omega}$. Here, we say a map from a sheaf $F$ to the degree-zero term of a cosimplicial sheaf $C$ is a cosimplicial resolution if it yields an exact complex

$$
F \rightarrow s(C) \text {. }
$$

The lemma is saying that

$$
\tilde{\omega} \rightarrow \tilde{\omega}_{X^{0}} \rightarrow \tilde{\omega}_{X^{1}} \rightarrow \cdots
$$

is exact.

We once more remind the reader that in proofs like that of the previous lemma, one should actually be arguing level-by-level, e.g. with the rings $W_{m}\left[t_{1}, t_{2}, \ldots, t_{n}\right]$.

Lemma 12. Denote by $C\left(W \tilde{\omega}_{X}\right)$ the cosimplical CDGA on $Y$ associated to the collection $W \tilde{\omega}_{X^{i}}:=$ $H\left(\tilde{\omega} \otimes_{\mathcal{O}_{Z}} \mathcal{O}_{X^{i}}\right)$, and let $s\left(C\left(W \tilde{\omega}_{X}\right)\right)$ be the associated simple complex. So this is the simple complex associated to the double complex

$$
W \tilde{\omega}_{X^{0}} \rightarrow W \tilde{\omega}_{X^{1}} \rightarrow \cdots
$$

Then

$$
W \tilde{\omega} \rightarrow s\left(C\left(W \tilde{\omega}_{X}\right)\right)
$$

is a quasi-isomorphism.

Proof. Let $J_{i}$ again be the locally principal ideal defining $X_{i}$ in $Z$ and $J=\prod J_{i}$.

First, we note that the (inverse) Cartier isomorphism for $\Omega_{Z}(\log E) \bigotimes_{W} k$ induces one on $\tilde{\omega}_{X^{i}} \bigotimes_{k}$ for each $i$. To see this, note the following diagram obtained from that of the previous corollary by forming a tensor with $\Omega_{Z}(\log E)$ :

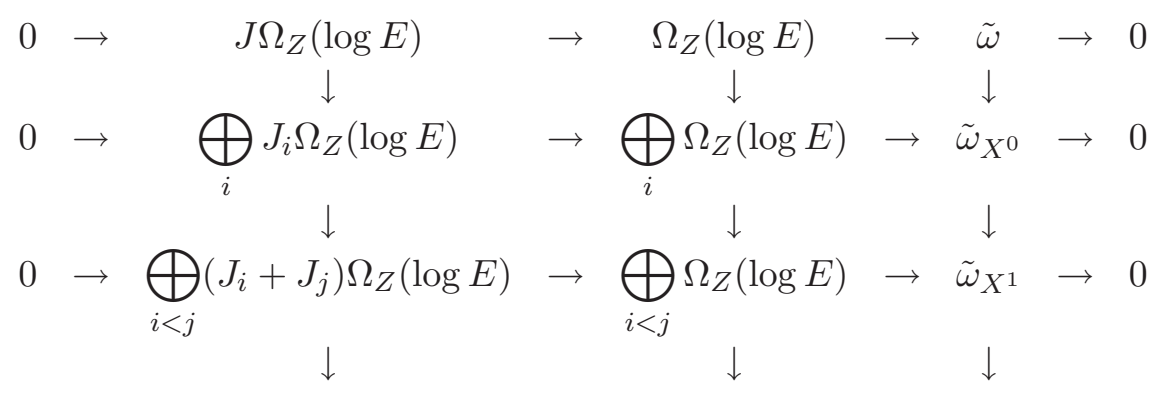

The horizontal sequences are short exact and remain so after reducing mod $p$. So we need only show that the Cartier isomorphism on $\Omega_{Z}(\log E) \bigotimes_{W} k$ induces one on $J \Omega_{Z}(\log E) \bigotimes_{W} k$ and $\left(J_{i_{1}}+\cdots+J_{i_{l}}\right) \Omega_{Z}(\log E) \otimes k$ for each collection of indices $i_{1}, \ldots, i_{l}$.

One sees this for the complex $J_{i} \Omega_{Z}(\log E) \otimes k$ by writing it étale locally as the tensor product of the complexes

$$
\begin{gathered}
\left(t_{i}\right) \rightarrow k\left[t_{i}\right] d t_{i}, \\
k\left[t_{j}\right] \rightarrow k\left[t_{j}\right] d t_{j} / t_{j},
\end{gathered}
$$

for $1 \leqslant j \leqslant b, j \neq i$, and

$$
k\left[t_{j}\right] \rightarrow k\left[t_{j}\right] d t_{j}
$$

for $j>b$, noting that the Cartier isomorphism holds for each term separately (the only new computation is for the first complex), and using the Künneth formula. Exactly the same argument implies the Cartier isomorphism for the cohomology of the complex

$$
J_{i_{1}} \cdots J_{i_{m}} \Omega_{Z}(\log E) \otimes k
$$




\section{KIM AND R. M. HAIN}

for any collection of indices, and hence for $J \Omega_{Z}(\log E) \otimes k$. For the complex $\left(J_{i}+J_{j}\right) \Omega_{Z}(\log E) \otimes k$ we get the result by putting it into an exact sequence

$$
0 \rightarrow J_{i} J_{j} \Omega_{Z}(\log E) \otimes k \rightarrow\left(J_{i} \Omega_{Z}(\log E) \oplus J_{j} \Omega_{Z}(\log E)\right) \otimes k \rightarrow\left(J_{i}+J_{j}\right) \Omega_{Z}(\log E) \otimes k \rightarrow 0
$$

and using the isomorphism for the first two terms. One can extend this argument to an arbitrary sum

$$
\left(J_{i_{1}}+\cdots+J_{i_{m}}\right) \Omega_{Z}(\log E) \otimes k
$$

by induction on the number of ideals. That is, the same argument as in the previous lemma and corollary gives us an exact sequence

$$
0 \rightarrow J_{i_{1}} \cdots J_{i_{m}} \rightarrow \bigoplus_{k} J_{i_{k}} \rightarrow \bigoplus_{k<l}\left(J_{i_{k}}+J_{i_{l}}\right) \rightarrow \cdots \rightarrow J_{i_{1}}+J_{i_{2}}+\cdots+J_{i_{m}} \rightarrow 0,
$$

and all the terms before the last involve $<m$ ideals. Since the inverse Cartier map is an isomorphism for each of them, it is an isomorphism for the last term.

Now we can repeat the definitions of [Hyo91, p. 245] verbatim to define the maps $\mathbf{p}: W_{n} \tilde{\omega}_{X} \rightarrow$ $W_{n+1} \tilde{\omega}_{X}$ and $\mathbf{p}: W_{n} \tilde{\omega}_{X^{i}} \rightarrow W_{n+1} \tilde{\omega}_{X^{i}}$ for each $i$ and the argument of [Hyo91, (2.2.2)] shows that they are injective. Furthermore, a repetition of the argument in [Hyo91, (2.4.1)] gives us that $W_{n+1} \tilde{\omega}_{X} / \mathbf{p}\left(W_{n} \tilde{\omega}_{X}\right)$ is QI to $\tilde{\omega}_{X} \bigotimes_{W} k$ and $W_{n+1} \tilde{\omega}_{X^{i}} / \mathbf{p}\left(W_{n} \tilde{\omega}_{X^{i}}\right)$ is quasi-isomorphic to $\tilde{\omega}_{X^{i}} \bigotimes_{W} k$. Furthermore, both $\mathbf{p}$ and the quasi-isomorphisms $W_{n+1} \tilde{\omega}_{X^{i}} / \mathbf{p}\left(W_{n} \tilde{\omega}_{X^{i}}\right) \simeq \tilde{\omega}_{X^{i}} \bigotimes_{W} k$ are compatible with the restrictions from the complexes on $X^{i}$ to that on $X^{i+1}$. That is, we have an exact sequence of double complexes:

$$
\begin{aligned}
& 0 \rightarrow W_{n} \tilde{\omega}_{X} \rightarrow W_{n+1} \tilde{\omega}_{X} \rightarrow W_{n+1} \tilde{\omega}_{X} / \mathbf{p}\left(W_{n} \tilde{\omega}_{X}\right) \rightarrow 0 \\
& \stackrel{\downarrow}{\downarrow} \underset{W_{n} \tilde{\omega}_{X^{0}}}{\downarrow} \rightarrow W_{n+1} \tilde{\omega}_{X^{0}} \rightarrow W_{n+1} \tilde{\omega}_{X^{0}} / \mathbf{p}\left(W_{n} \tilde{\omega}_{X^{0}}\right) \rightarrow 0
\end{aligned}
$$

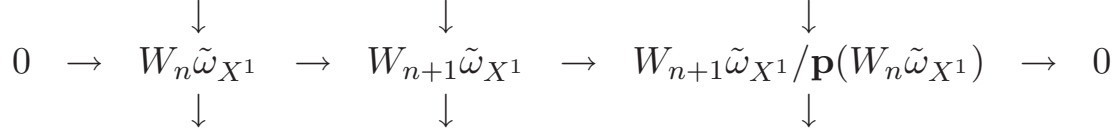

Therefore, the assertion of the lemma is reduced to the case of $n=1$, in which case it follows again from the Cartier isomorphism.

We will see below in the proof of independence of the weight filtration that this resolution also is independent of the admissible lift.

A proof identical to Mokrane's shows that

$$
P_{j}^{\prime} W \tilde{\omega}_{X^{i}}:=\underline{H}\left(\left(P_{j}^{\prime}\left(\tilde{\omega} \otimes \mathcal{O}_{X^{i}}\right)\right)\right),
$$

where the $P^{\prime}$ inside the cohomology is again the image of the filtration on $\Omega_{Z}(\log E)$, injects into $W \tilde{\omega}_{X^{i}}$. If one tensors the short exact sequence in [Mok93, Lemme 1.2] with $\mathcal{O}_{D_{i}}$ where $D_{i}$ is one of the components of the divisor over $t=0$ (which in our notation would be one of the $X_{i}$ ), one gets

$$
\begin{aligned}
& 0 \rightarrow P_{j-1}^{\prime}\left(\Omega_{Z / S}(\log (D)) \bigotimes_{\mathcal{O}_{Z}} \mathcal{O}_{D_{i}}\right) \rightarrow P_{j}^{\prime}\left(\Omega_{Z / S}(\log (D)) \bigotimes_{\mathcal{O}_{Z}} \mathcal{O}_{D_{i}}\right) \\
& \rightarrow G r_{j}^{P^{\prime}} \Omega_{Z / S}(\log (D)) \bigotimes_{\mathcal{O}_{Z}} \mathcal{O}_{D_{i}} \rightarrow 0 .
\end{aligned}
$$

Then the retraction used in the proof works equally well for this sequence to show that the coboundary maps of the associated cohomology sequence are zero.

For any fixed set $I$ of indices, the filtration $P_{j}^{\prime}$ on $\tilde{\omega}_{X_{I}}$ is the convolution of an 'internal' weight filtration $P^{\prime}(I)$ involving log poles coming from components $X_{i}$ for $i \in I$, and an 'external' filtration $P^{\prime}(E)$ coming from contributions of $X_{k}$ for $k \notin I$ and the horizontal divisors $H_{l}$. Hence, on all 


\section{Crystalline Rational homotopy theory}

of $\tilde{\omega}_{X^{i}}$ we can break up the filtration into these two parts. The graded pieces for the two filtrations are calculated separately using the residue formula as usual:

$$
G r_{l}^{P^{\prime}(I)} \tilde{\omega}_{X^{i}}=\omega_{X^{i}}^{\left(\begin{array}{c}
i+1 \\
l
\end{array}\right)}[-l] .
$$

On the other hand, for each indexing set $I \subset\{1, \ldots, a\}$, we have

$$
G r_{k}^{P^{\prime}(E)} \omega_{X_{I}}=\bigoplus_{J \subset\{1, \ldots, b\}-I,|J|=k} \Omega_{X_{I \cup J} / S}[-k],
$$

so we get that

$$
G r_{k}^{P^{\prime}(E)} \omega_{X^{i}} \simeq \bigoplus_{K} \Omega_{X_{K}}[-k]
$$

for various indexing sets $K$ of cardinality $i+1+k$ (allowing multiplicities). Therefore, combining the convolution formula for the graded pieces together with the injectivity above, we see that the terms

$$
G r_{j}^{P^{\prime}} W \tilde{\omega}_{X^{i}}
$$

are isomorphic to a direct sum of $W \omega_{E_{T}}[-j](-j)$ for indexing sets $T$ of cardinality $i+1+k$ where $k$ runs between 0 and $j$. Note here that the $W \omega_{E_{T}}$ are De Rham-Witt complexes for smooth varieties (without log structures).

We can also follow Mokrane essentially literally to show that the weight filtration $P^{\prime}$ on each $W \tilde{\omega}_{X^{i}}$ is independent of the admissible lifting. For this reason, we just give a brief sketch: If $(Z, X, E)$ and $\left(Z^{\prime}, X^{\prime}, E^{\prime}\right)$ are two different lifts, the comparison is effected by constructing the blow-up of $Z \times_{\operatorname{Spec}(W)} Z^{\prime}$ along the subscheme $\sum E_{i} \times E_{i}^{\prime}$ and removing the strict transforms of each $Z \times E_{i}^{\prime}$ and $E_{i} \times Z^{\prime}$. Denote the resulting scheme by $Z^{\prime \prime}$ and the exceptional divisor by $E^{\prime \prime}$. Denote by $X^{\prime \prime}$ the total transform of just the vertical components $X_{i} \times X_{i}^{\prime}$. Finally, let $Y^{\prime \prime}$ be the intersection with $Z^{\prime \prime}$ of the total transform of $Y$, embedded diagonally in $Z \times Z^{\prime}$. Thus, the local picture looks as follows:

$$
\begin{aligned}
Z=\operatorname{Spec} W\left[t_{1}, \ldots, t_{n}\right], & Z^{\prime}=\operatorname{Spec} W\left[t_{1}^{\prime}, \ldots, t_{n}^{\prime}\right], \\
X=\operatorname{Spec} W\left[t_{1}, \ldots, t_{n}\right] /\left(t_{1} \cdots t_{a}\right), & X^{\prime}=\operatorname{Spec} W\left[t_{1}^{\prime}, \ldots, t_{n}^{\prime}\right] /\left(t_{1}^{\prime} \cdots t_{a}^{\prime}\right) .
\end{aligned}
$$

Here $E$ is given by $t_{1} \cdots t_{b}=0$ and $E^{\prime}$ by $t_{1}^{\prime} \cdots t_{b}^{\prime}=0$ for some $b \geqslant a$. Then

$$
\begin{aligned}
Z^{\prime \prime} & =\operatorname{Spec} W\left[t_{1}, \ldots, t_{n}, t_{1}^{\prime}, \ldots, t_{n}^{\prime}, v_{1}^{ \pm 1}, \ldots, v_{b}^{ \pm 1}\right] /\left(t_{1}^{\prime}-v_{1} t_{1}, \ldots, t_{b}^{\prime}-v_{b} t_{b}\right) \\
& =\operatorname{Spec} W\left[t_{1}, \ldots, t_{n}, t_{b+1}^{\prime}, \ldots, t_{n}^{\prime}, v_{1}^{ \pm 1}, \ldots, v_{b}^{ \pm 1}\right] .
\end{aligned}
$$

The exceptional divisor $E^{\prime \prime}$ is given by

$$
t_{1} \cdots t_{b}=\left(\prod v_{i}\right) \prod t_{i}^{\prime}=0
$$

$X^{\prime \prime}$ by

$$
t_{1} \cdots t_{a}=\left(\prod_{i=1}^{a} v_{i}\right) \prod t_{i}^{\prime}=0
$$

while $Y^{\prime \prime}$ is given by the ideal

$$
\left(t_{i}-t_{i}^{\prime}, t, p\right)_{i=1, \ldots, n}
$$

Let $\mathcal{D}^{\prime}$ be a pro-sheaf consisting of the divided power envelope of $Y^{\prime \prime}$ in $Z^{\prime \prime}$ and let $J$ (respectively $J^{\prime}$ ) be the smallest sub-PD ideal of $\mathcal{D}^{\prime}$ containing the ideal defining $Y$ in $Z$ (respectively $Z^{\prime}$ ); then $\mathcal{D}^{\prime} / J=\mathcal{D}^{\prime} / J^{\prime}$ and we denote that quotient by $\mathcal{D}^{\prime \prime}$. So $\mathcal{D}^{\prime \prime}$ locally looks like

$$
\mathcal{O}_{Z^{\prime \prime}} \bigotimes_{W\left[\phi_{1}, \ldots, \phi_{b}, \tau_{b+1}, \ldots, \tau_{n}\right]} W\left\langle\phi_{1}, \ldots, \phi_{b}, \tau_{b+1}, \ldots, \tau_{n}\right\rangle,
$$

where $y_{i}=v_{i}-1$ and $\tau_{i}=t_{i}-t_{i}^{\prime}$. 


\section{KIM AND R. M. HAIN}

We wish to compare cosimplicial resolutions of the three pro-sheaves $\tilde{\omega}_{X}, \tilde{\omega}_{X}^{\prime}$ and

$$
\tilde{\omega}_{X^{\prime \prime}}=\Omega_{Z^{\prime \prime} / W}\left(\log E^{\prime \prime}\right) \otimes \mathcal{O}_{X^{\prime \prime}} .
$$

If we give the last sheaf as well the filtration $P^{\prime}$ induced by the filtration on $\Omega_{Z^{\prime \prime} / W}\left(\log E^{\prime \prime}\right)$ by the number of log terms, the above local description shows that we have natural inclusions

$$
P_{j}^{\prime} \tilde{\omega}_{X} \hookrightarrow P_{j}^{\prime} \tilde{\omega}_{X^{\prime \prime}} \bigotimes_{\mathcal{O}_{Z}} \mathcal{D}^{\prime \prime} \hookleftarrow P_{j}^{\prime} \tilde{\omega}_{X^{\prime}}
$$

and hence also inclusions

$$
P_{j}^{\prime} \tilde{\omega}_{X^{i}} \hookrightarrow P_{j}^{\prime} \tilde{\omega}_{\left(X^{\prime \prime}\right)^{i}} \otimes \mathcal{D}^{\prime \prime} \hookleftarrow P_{j}^{\prime} \tilde{\omega}_{\left(X^{\prime}\right)^{i}}
$$

where the superscripts on $X^{\prime}$ and $X^{\prime \prime}$ are the obvious ones referring to tensor products with the structure sheaves of $i$-fold intersections of the components of $X^{\prime}$ and $X^{\prime \prime}$. But as in [Mok93], the local description gives us

$$
P_{j}^{\prime} \tilde{\omega}_{\left(X^{\prime \prime}\right)^{i}} \otimes \mathcal{D}^{\prime \prime} \simeq P_{j}^{\prime} \tilde{\omega}_{X^{i}} \bigotimes_{W} \Omega_{W\left\langle\phi_{1}, \ldots, \phi_{b}, \tau_{b+1}, \ldots, \tau_{n}\right\rangle / W}
$$

and

$$
W \simeq \Omega_{W\left\langle\phi_{1}, \ldots, \phi_{b}, \tau_{b+1}, \ldots, \tau_{n}\right\rangle / W}
$$

so the first inclusion is a QI and similarly for the second inclusion. If $U$ is the open subscheme of $Y$ obtained by removing the singular points as well as intersections with the horizontal divisor $H$, the cohomology sheaves of all three complexes can be viewed as subsheaves of the complex $W \tilde{\omega}_{U}$ from $[$ HK94, $\S 1.4]$. Thus, as explained by Jannsen in the appendix to [Hyo91], the independence of the filtration follows (that is, no cocycle condition needs to be checked).

Define a new cosimplicial sheaf $C(W \tilde{\omega}[u])$ by adjoining to $C(W \tilde{\omega})$ in each cosimplicial degree variables $u^{[i]}$ subject to the condition that $u[0]=1, d u^{[i]}=d t / t u^{[i-1]}$, each having weight two and Frobenius action $F\left(u_{i}\right)=p^{i} u_{i}$. Thus, $C(W \tilde{\omega}[u])$ gives a cosimplicial resolution of $W \tilde{\omega}[u]$, that is, there is a $\mathrm{QI}$ of complexes

$$
W \tilde{\omega}[u] \simeq s(C(W \tilde{\omega}[u]))
$$

and hence, a QI of CDGAs

$$
T W(C(W \tilde{\omega}[u])) \simeq T W(W \tilde{\omega}[u])
$$

On the other hand, we have a natural $\mathrm{QI} W \tilde{\omega}[u] \simeq W \omega$, and hence, a $\mathrm{QI}$

$$
T W(C(W \tilde{\omega}[u])) \simeq T W(W \omega) .
$$

We give to $C(W \tilde{\omega}[u])$ and to $s(C(W \tilde{\omega}[u]))$ the weight filtration $P$ defined by the convolution of the weight filtration on $W \tilde{\omega}[u]$ with the filtration by cosimplicial degree. That is,

$$
\begin{aligned}
P_{n}\left(W \tilde{\omega}_{X^{i}}[u]\right) & =P_{n+i}^{\prime}\left(W \tilde{\omega}_{X^{i}}[u]\right) \\
& =\bigoplus_{j} P_{n+i-2 j}^{\prime}\left(W \tilde{\omega}_{X^{i}}\right) u^{[j]} .
\end{aligned}
$$

By the independence of $P^{\prime}$ from the lifting, the same is true for $P$.

As in [Hai87b] it is easy to construct a QI from $s(C(W \tilde{\omega}[u]))$ to a cosimplicial resolution of the Hyodo-Steenbrink complex which is filtration preserving. This is achieved by concatenating our QI $W \tilde{\omega}[u] \rightarrow W \omega$ with Mokrane's $W \omega \rightarrow W A$ at each level. Therefore, on cohomology, we get a filtration-preserving isomorphism. In the case that $k$ is a finite field, we will see below that the cohomology of $s(C(W \tilde{\omega}[u]))$ is a mixed isocrystal as was shown for the cohomology of $W A$ by Mokrane, so a simple strictness argument shows that the two filtrations agree on cohomology.

On the other hand, $P$ induces a natural filtration on $T W(C(W \tilde{\omega}[u]))$, and hence on $B(Y, y)$ and $\operatorname{Cr}(Y, y)$. 


\section{Crystalline RATional homotopy THEORY}

Theorem 3. Suppose the field $k$ is finite. Then $(T W(W \omega), T W(C(W \tilde{\omega}[u])), P)$ is a commutative mixed Frobenius algebra.

Proof. As filtered complexes, $T W(C(W \tilde{\omega}[u]))$ is filtered QI to

$$
R \Gamma(s(C(W \tilde{\omega}[u]))),
$$

so we may compute the terms of the spectral sequence with the latter. We compute the $E_{0}$ term:

$$
\begin{aligned}
E_{0}^{-n, q} & =G r_{n}[R \Gamma(s(C(W \tilde{\omega}[u])))]^{q-n} \\
& =\bigoplus_{0 \leqslant t \leqslant n} G r_{n}\left[R \Gamma\left(W \tilde{\omega}_{X^{t}}[u]\right)\right]^{q-n-t} \\
& =\bigoplus_{0 \leqslant t \leqslant n} G r_{n+t}^{P^{\prime}}\left[R \Gamma\left(W \tilde{\omega}_{X^{t}}[u]\right)\right]^{q-n-t} \\
& =\bigoplus_{0 \leqslant t \leqslant n} \bigoplus_{i} G r_{n+t-2 i}^{P^{\prime}}\left[R \Gamma\left(W \tilde{\omega}_{X^{t}}\right)\right]^{q-n-t}(-i) \\
& =\bigoplus_{0 \leqslant t \leqslant n} \bigoplus_{i}\left[G_{i}[-n-t+2 i](-n-t+2 i)\right]^{q-n-t}(-i) \\
& =\bigoplus_{0 \leqslant t \leqslant n} \bigoplus_{i} G_{i}^{q-2 n-2 t+2 i}(-n-t+i),
\end{aligned}
$$

where $G_{i}$ is a direct sum of complexes $R \Gamma\left(W \omega_{E_{T}}\right)$ for some collection of subsets $T \subset\{1, \ldots b\}$ of cardinality $\geqslant t$. Thus,

$$
E_{1}^{-n, q}=\bigoplus_{t, i} H^{q-2 n-2 t+2 i}\left(G_{i}\right)(-n-t+i)
$$

which is pure of weight $q$, being built out of the crystalline cohomology of proper smooth varieties. This finishes the proof.

Now given a point $y \in Y$, we get augmentations of $T W(C(W \tilde{\omega}[u]))$ and $T W\left(W \omega_{Y}\right)$. Using them, we can form the bar complexes

$$
B\left(T W(C(W \tilde{\omega}[u])), a_{y}\right)
$$

and $B(Y, y)$ which are quasi-isomorphic. Thus, the weight filtration on

$$
H\left(B\left(T W(C(W \tilde{\omega}[u])), a_{y}\right)\right)
$$

induces one on $H(B(Y, y))$ which is compatible with the Hopf algebra structure. Therefore, we get a weight filtration on Lie $\pi_{1}^{\mathrm{cr}}(Y, y)=\left(Q H^{0}(B(Y, y))\right)^{*}$.

The previous theorem says that if $k$ is finite, then

$$
\left(T W\left(W \omega_{Y}\right), T W(C(W \tilde{\omega}[u])), P\right)
$$

is a mixed Frobenius algebra. Therefore, we get the structure of a mixed $F$-isocrystal on $\operatorname{Lie} \pi_{1}^{\mathrm{cr}}(Y, y)$ as well.

\section{Comparison with the De Rham fundamental group: proof of Theorem 2}

Now suppose $X$ and $Y$ are as in Theorem 2. Denote by $\hat{X}$ the formal completion of $X$ along $Y$. Regard all of the objects as log (formal) schemes with the log structure coming from the horizontal divisor $D$. Thus, $X, \hat{X}$ and $Y$ are all smooth with respect to the trivial log structures on $W$ and $k$. 


\section{KIM AND R. M. HAIN}

We would like to compare the crystalline fundamental group of $Y$ with the De Rham fundamental group of $X^{*}$, the generic fiber of $X$. At the level of cohomology, this is the Berthelot-Ogus theorem [BO83] and we need only to take care that the appropriate maps are multiplicative. To do this, we need to make explicit several maps in that paper that are only defined in the derived category by defining explicit maps between embedding systems that realize them.

In the proof, we will use the computation of the crystalline fundamental group via the crystalline complex associated to an embedding system, as explained in $\S 4$. As a general remark, it is important to note that the comparison isomorphism between the crystalline complexes associated to two different embedding systems is effected via a third embedding system dominating both, and the associated inclusion map of the crystalline complexes is via pull-back of differential forms. Hence, the crystalline complexes are actually quasi-equivalent as algebras.

We start with the following preliminary lemma.

LEMMA 13. Let

$$
Y \rightarrow S
$$

be a map of fine saturated log schemes over $k$ and let $S \hookrightarrow T$ be an exact $P D$-immersion. Suppose $T$ admits a Frobenius lift. Then there exists an embedding system $(Y, Z$.) which admits a Frobenius lift compatible with the Frobenius of $T$.

Proof. The proof is analogous to [Ill79, p. 602]. Let $\cup_{i} U_{i}$ be an affine open covering of $Y$ such that each $U_{i}$ lifts to a smooth formal $T$-log scheme $\tilde{U}_{i}$ (these exist by [Kat89, Proposition 3.14]). Let $U=\coprod U_{i}, \tilde{U}=\coprod \tilde{U}_{i}$. Then the Frobenius $f$ from $U$ to $U$ lifts to $\tilde{U} \rightarrow \tilde{U}$ by smoothness, in a manner compatible with the Frobenius of $T$. On the other hand, $U \rightarrow Y$ is a morphism of cohomological 2-descent for sheaves having any fixed $n$-torsion in the étale topology [Sai72, Corollary 4.3.5]. This means that for the sheaves we are considering (pro-systems over $n$ of $p^{n}$-torsion sheaves) this covering extends (via taking the coskeleton) to a simplicial hypercovering $Y$. $\rightarrow Y$ such that $Y_{i}=U \times_{Y} U \times_{Y} \cdots \times_{Y} U(i+1$ times $)$. On the other hand, each $Y_{i}$ embeds naturally into an $(i+1)$-fold product of $U$ over $S$ which, in turn, embeds into $Z_{i}:=\tilde{U} \times_{T} \tilde{U} \times_{T} \cdots \times_{T} \tilde{U}$. The Frobenius lifting of $Z_{1}=\tilde{U}$ induces product maps $Z_{i} \rightarrow Z_{i}$, which give us the required lifting.

Choose a uniformizer $\pi$ of $A$ which therefore determines a presentation $A \simeq W[t] /(f(t))$, where $f(t)$ is an Eisenstein polynomial of degree $e=[F: K]$. Let $R$ be the $p$-adic completion of the divided power envelope of $(f(t), p)$ inside $W[t]$. Thus $R$ is also the DP envelope of the ideal $\left(t^{e}\right)$. We have a natural map $g: R \rightarrow W\langle\langle t\rangle\rangle$. On the other hand, if $f$ denotes the Frobenius map on $W\langle\langle t\rangle\rangle$ which is the usual Frobenius on $W$ and the $p$ th power map on $t$ and $r$ is such that $p^{r} \geqslant e$, then the map $\phi^{r}: W\langle\langle t\rangle\rangle \rightarrow W\langle\langle t\rangle\rangle$ factors through $W\langle\langle t\rangle\rangle \rightarrow R \rightarrow W\langle\langle t\rangle\rangle$. Note that the Frobenius map defined above also induces a Frobenius map $R \rightarrow R$. Let $Y^{\prime}=X \otimes A / p$ with the induced log structure. The Berthelot-Ogus isomorphism hinges on the comparison between the crystalline cohomology of $Y$ with respect to $W\langle\langle t\rangle\rangle$ and $Y^{\prime}$ with respect to $R$. That is, we have the commutative diagram

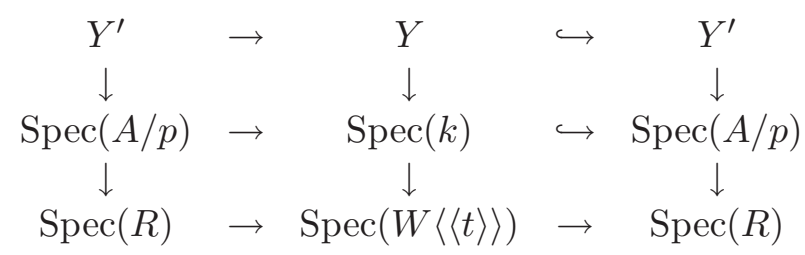

where the composite of the horizontal arrows are all the $r$ th iterate of the Frobenius. We choose crystalline complexes for $Y$ and $Y^{\prime}$ as follows. Construct first embedding systems for $Y$ and $Y^{\prime}$ with 


\section{CRYstalline RATional HOMOTOPY THEORY}

respect to $W[t]$ which fit into a diagram

$$
\begin{array}{cccccc}
Y . & \hookrightarrow & Y^{\prime} & \hookrightarrow & Z . \\
\downarrow & & \downarrow & & \\
Y & & & Y^{\prime} & &
\end{array}
$$

where $Z$. is smooth over $W[t]$ and the left-hand square is cartesian. We can also arrange for $Z$. to admit a Frobenius lift compatible with the Frobenius of $W[t]$. Then $C=\Omega_{Z / W[t]} \otimes W\langle\langle t\rangle\rangle$ and $C^{\prime}=\Omega_{Z / W[t]} \otimes R$ are crystalline complexes for $Y$ and $Y^{\prime}$ and we can regard both as simplicial sheaves of CDGAs on $Y$.

Denote by $C^{(r)}$ (respectively $\left(C^{\prime}\right)^{(r)}$ ) the pull-back of $C$ (respectively $C^{\prime}$ ) by the $r$ th power of the Frobenius map of $W\langle\langle t\rangle\rangle$ (respectively $R$ ). Then the big diagram above implies that

$$
\left(C^{\prime}\right)^{(r)} \simeq C^{(r)} \bigotimes_{W\langle\langle t\rangle\rangle} R
$$

in the homotopy category of sheaves of CDGAs on $Y$. On the other hand, the Frobenius lifts induce maps $C^{(r)} \rightarrow C$ and $\left(C^{\prime}\right)^{(r)} \rightarrow C^{\prime}$. So we have maps of sheaves of CDGAs

$$
C \otimes R \leftarrow C^{(r)} \otimes R \simeq\left(C^{\prime}\right)^{(r)} \rightarrow C^{\prime}
$$

and taking Thom-Whitney algebras, we have multiplicative maps

$$
T W(C) \otimes(R \otimes \mathbf{Q}) \leftarrow T W\left(C^{(r)}\right) \otimes(R \otimes \mathbf{Q}) \simeq T W\left(\left(C^{\prime}\right)^{(r)}\right) \rightarrow T W\left(C^{\prime}\right)
$$

of CDGAs over $R \otimes \mathbf{Q}$. The theorem of Berthelot and Ogus implies that all the maps are quasiequivalences (since the usual maps involving the simple complexes rather than $T W$ algebras are quasi-isomorphisms). Now we form a tensor with the quotient map $R \otimes \mathbf{Q} \rightarrow A \otimes \mathbf{Q}=F$ to get

$$
T W(C) \bigotimes_{W\langle\langle t\rangle\rangle \otimes \mathbf{Q}} F \simeq T W\left(C^{\prime}\right) \bigotimes_{R \otimes Q} F .
$$

By using the fact that $C^{\prime} \otimes A$ is the crystalline complex associated to an embedding system for $Y^{\prime}$ with respect to $A$ which is also true of $\Omega_{\hat{X} / A}$, we get

$$
T W\left(C^{\prime}\right) \otimes F \simeq T W\left(\Omega_{\hat{X} / A}\right) \simeq T W\left(\Omega_{X^{*} / F}\right),
$$

where the last quasi-equivalence follows from formal GAGA. On the other hand, $C$ is quasiequivalent to the base change to $W\langle\langle t\rangle\rangle$ of a crystalline complex for $Y$ with respect to $W$ so we get

$$
T W(C) \otimes F \simeq T W\left(W \omega_{Y}\right) \bigotimes_{K} F
$$

giving us the desired quasi-equivalence

$$
T W\left(W \omega_{Y}\right) \bigotimes_{K} F \simeq T W\left(\Omega_{X * / F}\right) .
$$

This gives the isomorphism of homotopy types stated in the theorem.

It is straightforward to check that this equivalence is compatible with base-points. So putting everything together, we see that we get a quasi-equivalence of augmented algebras

$$
\left(T W\left(\Omega_{X^{*} / K}\right), x\right) \simeq\left(T W\left(W \omega_{Y /(W, W(L))}, y\right)\right) \bigotimes_{K} F
$$

and therefore

$$
C r(Y, y) \bigotimes_{K} F \simeq D R\left(X^{*}, x\right)
$$

of the theorem, an isomorphism of commutative Hopf algebras over $K$. 


\section{KIM AND R. M. HAIN}

As proved in [Woj93], this last object is the coordinate ring of the De Rham fundamental group of $X^{*}$ and the isomorphism is clearly compatible with the bar filtration (since it is induced by a QI at the level of augmented CDGAs), so we conclude that

$$
\pi_{1}^{\mathrm{cr}}(Y, y) \bigotimes_{K} F \simeq \pi_{1}^{\mathrm{dr}}\left(X^{*}, x\right)
$$

Although we are concentrating on the fundamental group for this paper, the higher cohomology of the bar complex is also of interest. In particular, if the varieties are simply connected, they can be used to define higher crystalline rational homotopy groups.

Proof of Corollary 2. The isomorphism classes of the higher rational homotopy groups are determined by their dimension, and this dimension can be computed in any complex embedding of $F$ or after base change to the completion $F_{v}$ of $F$ with respect to $v$. The assumptions imply that the special fibers $Y$ and $Y^{\prime}$ are isomorphic smooth $\log$ schemes. Thus, $T W\left(\omega_{Y}\right) \simeq T W\left(\omega_{Y^{\prime}}\right)$, which implies the quasi-equivalence of $T W\left(\Omega_{X}(\log D)\right) \otimes F_{v}$ and $T W\left(\Omega_{X}^{\prime}\left(\log D^{\prime}\right)\right) \otimes F_{v}$. Thus, their bar complexes are quasi-equivalent, giving isomorphisms of their cohomology groups, i.e. the higher De Rham homotopy groups of $U$ and $U^{\prime}$ [Woj93].

In the projective case without divisors this theorem follows from Artin and Mazur's étale homotopy theory [AM86] where a stronger integral statement is proved. The rational statement in the smooth proper case without divisors can also be deduced from the formality theorem of Deligne et al. [DGMS75] together with the proper base change theorem for étale cohomology and the comparison theorem between étale and Betti cohomologies over $\mathbb{C}$.

\section{ACKNOWLEDGEMENTS}

MK is grateful to Arthur Ogus for several useful discussions related to the comparison theorem and to Kirti Joshi for numerous conversations on $p$-adic Hodge theory. He is also grateful to the Korea Institute for Advanced Study for providing the environment where the revisions to this paper could be carried out.

Two referees of earlier versions of this paper gave it unusually thorough readings and made many helpful suggestions for improving the proofs and exposition. For this we are extremely grateful.

Both authors were supported in part by grants from the National Science Foundation.

\section{REFERENCES}

AM86 M. Artin and B. Mazur, Etale homotopy, reprint of the 1969 original, Lecture Notes in Mathematics, vol. 100 (Springer, Berlin, 1986).

Blo77 S. Bloch, Algebraic K-theory and crystalline cohomology, Publ. Math. Inst. Hautes Études Sci. 47 (1977), 187-268.

BO83 P. Berthelot and A. Ogus, F-isocrystals and de Rham cohomology. I, Invent. Math. 72 (1983), 159-199.

Che77 K. T. Chen, Iterated path integrals, Bull. Amer. Math. Soc. 83 (1977), 831-879.

CLS99 B. Chiarellotto and B. Le Stum, F-isocristaux unipotents, Compositio Math. 116 (1999), 81-110.

Del74 P. Deligne, Théorie de Hodge. III, Publ. Math. Inst. Hautes Études Sci. 44 (1974), 5-77.

Del89 P. Deligne, Le groupe fondamental de la droite projective moins trois points, in Galois groups over Q (Berkeley, CA, 1987), Math. Sci. Res. Inst. Publ., vol. 16 (Springer, New York, 1989), 79-297.

DGMS75 P. Deligne, P. Griffiths, J. Morgan and D. Sullivan, Real homotopy theory of Kähler manifolds, Invent. Math. 29 (1975), 245-274. 


\section{CRYstalline RATional HOMOTOPY THEORY}

Fal90 G. Faltings, F-isocrystals on open varieties: results and conjectures, in The Grothendieck Festschrift, vol. II, Progr. Math., vol. 87 (Birkhäuser, Boston, MA, 1990), 219-248.

God58 R. Godement, Topologie algébrique et théorie des faisceaux, Actualités Sci. Indust., vol. 1252, Publ. Math. Univ. Strasbourg, vol. 13 (Hermann, Paris, 1958).

Hai87a R. M. Hain, The de Rham homotopy theory of complex algebraic varieties. I, K-Theory 1 (1987), $271-324$.

Hai87b R. M. Hain, The de Rham homotopy theory of complex algebraic varieties. II, K-Theory 1 (1987), 481-497.

Hai98 R. M. Hain, The Hodge de Rham theory of relative Malcev completion, Ann. Sci. École Norm. Sup. (4) 31 (1998), 47-92.

HK94 O. Hyodo and K. Kato, Semi-stable reduction and crystalline cohomology with logarithmic poles, in Périodes p-adiques (Bures-sur-Yvette, 1988), Astérisque 223 (1994), 221-268.

Hyo88 O. Hyodo, A cohomological construction of Swan representations over the Witt ring. I, Proc. Japan Acad. Ser. A Math. Sci. 64 (1988), 300-303.

Hyo91 O. Hyodo, On the de Rham-Witt complex attached to a semi-stable family, Compositio Math. 78 (1991), 241-260.

Ill79 L. Illusie, Complexe de de Rham-Witt et cohomologie cristalline, Ann. Sci. École Norm. Sup. (4) 12 (1979), 501-661.

Kat89 K. Kato, Logarithmic structures of Fontaine-Illusie, in Algebraic analysis, geometry, and number theory (Baltimore, MD, 1988) (Johns Hopkins Univ. Press, Baltimore, MD, 1989), 191-224.

Mac98 S. MacLane, Categories for the working mathematician, second edition, Graduate Texts in Mathematics, vol. 5 (Springer, New York, 1998).

Mok93 A. Mokrane, La suite spectrale des poids en cohomologie de Hyodo-Kato, Duke Math. J. 72 (1993), 301-337.

Mor78 J. W. Morgan, The algebraic topology of smooth algebraic varieties, Publ. Math. Inst. Hautes Études Sci. 48 (1978), 137-204.

Nak02 Y. Nakkajima, p-adic weight spectral sequences of log varieties, Preprint (2002).

Nav87 V. Navarro-Aznar, Sur la théorie de Hodge-Deligne, Invent. Math. 90 (1987), 11-76.

Oda95 T. Oda, A note on ramification of the Galois representation on the fundamental group of an algebraic curve. II, J. Number Theory 53 (1995), 342-355.

Ogu95 A. Ogus, F-crystals on schemes with constant log structure, Compositio Math. 97 (1995), 187-225.

Qui69 D. Quillen, Rational homotopy theory, Ann. of Math. (2) 90 (1969), 205-295.

Sai72 B. Saint-Donat, Techniques de descent cohomologique, in Séminaire de géométrie algébrique 4, Lecture Notes in Mathematics, vol. 270, part 2 (Springer, Berlin, 1972), ch. V.

Shi98 A. Shiho, Crystalline fundamental groups and p-adic Hodge theory, in The arithmetic and geometry of algebraic cycles (Banff, AB, 1998), CRM Proc. Lecture Notes, vol. 24 (Amer. Math. Soc., Providence, RI, 2000), 381-398.

Shi00 A. Shiho, Crystalline fundamental groups. I, in Isocrystals on log crystalline site and log convergent site, J. Math. Sci. Univ. Tokyo 7 (2000), 509-656.

Shi02 A. Shiho, Log convergent cohomology and rigid cohomology, J. Math. Sci. Univ. Tokyo 9 (2002), $1-163$.

Sul77 D. Sullivan, Infinitesimal computations in topology, Publ. Math. Inst. Hautes Études Sci. 47 (1977), 269-331 (1978).

Vol01 V. Vologodsky, Hodge structure on the fundamental group and its applications to p-adic integration, Preprint (2001), math.AG/0108109.

Woj93 Z. Wojtkowiak, Cosimplicial objects in algebraic geometry, in Algebraic K-theory and algebraic topology (Lake Louise, AB, 1991), NATO Adv. Sci. Inst. Ser. C Math. Phys. Sci., vol. 407 (Kluwer, Dordrecht, 1993), 287-327.

Zuc85 S. Zucker, Variation of mixed Hodge structures. II, Invent. Math. 80 (1985), 543-565. 


\section{CRYstalline RATional homotopy THEORY}

Minhyong Kim kim@math.arizona.edu

Department of Mathematics, University of Arizona, Tucson, AZ 85721, USA

and

Korea Institute For Advanced Study, 207-43 Cheongryangri-Dong, Dongdaemun-Gu, Seoul, Korea

Richard M. Hain hain@math.duke.edu

Department of Mathematics, Duke University, Durham, NC 27708, USA 